\title{
NEW VIEWPOINTS IN THE GEOMETRY OF SUBMANIFOLDS OF $R^{N}$
}

\author{
BY ROBERT B. GARDNER ${ }^{1}$
}

0. Introduction. The geometry of submanifolds of euclidean space is the oldest branch of differential geometry. The subject was the original source of most of the classical and modern ideas in the field, and still is the setting in which seemingly complicated general phenomena are most easily understood. In fact as Allendoerfer [1] once said "... an excellent way of discovering [theorems] is first to consider an imbedded manifold and then later to invent a proof applicable to a general abstract manifold." In fact it is of ten true that a concept which can be defined for submanifolds of Riemannian manifolds may be introduced in a simpler and more natural way for submanifolds immersed in euclidean space. In this paper we will always try to adopt the second course.

The geometry of hypersurfaces, that is, codimension one immersions, is familiar ground to most mathematicians, but once the codimension is increased the material is neither as familiar nor as satisfactory. In fact rather mysterious concepts appear, such as E. Cartan's exterior orthogonality of a linear system of quadratic forms, and C. Allendoerfer's type number. The plan of this report is to systematically expose the basic invariants of arbitrary codimension immersions in a way we regard as algebraically and geometrically natural. The viewpoints are often not standard. New invariants such as curvature deficiency and the $t$ th-trace rank are introduced and applied. Some old invariants such as the Allendoerfer type number are given new geometric interpretations, and old applications are reexamined and refined. New methods such as the systematic exploitation of the minimal enveloping subspaces of linear systems in tensor and exterior products lead to new viewpoints in which the Gauss integrability conditions appear as a short exact sequence of linear systems. Our viewpoint is both algebraically and geometrically natural, the symbiotic effect of this double viewpoint will be illustrated through the Allendoerfer theory of type number and some recent results on reducibility of immersions.

An address delivered at the seven hundred seventeenth meeting of the American Mathematical Society in Nashville on November 8, 1974, by invitation of the Committee to Select Hour Speakers for Southeast Sectional Meetings; received August 15, 1975.

AMS (MOS) subject classifications (1970). Primary 53C40, 53B25, 53A55.

Key words and phrases. Levi-Civita connection, curvature matrix, vector valued second fundamental form, minimal enveloping subspace, curvature rank, curvature deficiency, $t$ th trace rank, curvature wedge length, osculation degree, Gauss sequence, Allendoerfer type number, reducible immersions, cylindrical immersions.

${ }^{1}$ Research partially supported by NSF Grant MPS-75-06498. 
I would like to thank M. Spivak for making available an early edition of Volumes 4 and 5 of his Comprehensive introduction to differential geometry, and thus considerably reducing the number of topics I might have covered. I would also like to thank my colleague P. Eberlein for making many suggestions, and for listening to this work from inception to completion.

1. Preliminary notations and conventions. In this paper we are interested in immersions $X: M_{m} \rightarrow R^{m+p}$ of $m$-manifolds into euclidean $(m+p)$-space.

In order to save writing we agree to use the following range of indices throughout the paper

$$
\overbrace{\text { tangential range }}^{1 \leqslant i, j, k \leqslant m ;} \quad m+1 \leqslant a, b, c \leqslant m+p ; \quad 1 \leqslant A, B, C \leqslant m+p .
$$

We shall also follow the convention that sums are over repeated indices with the ranges omitted where they are understood.

Let $M_{m}$ be a Riemannian manifold with Riemannian metric I. If $U \subset M_{m}$ is an open set then we can introduce a local orthonormal coframe of 1-forms $\left\{\tau^{j}\right\} \quad(1 \leqslant j \leqslant m)$ in terms of which $\left.\dot{\mathrm{I}}\right|_{U}=\sum\left(\tau^{i}\right)^{2}$. Such a local coframe gives rise to the Levi-Civita connection matrix, which is the matrix of 1-forms $\left\{\phi_{i}^{j}\right\}$ uniquely characterized by the equations

$$
d \tau^{i}=\Sigma \tau^{j} \wedge \phi_{j}^{i} \text { and } \phi_{i}^{j}=-\phi_{j}^{i} .
$$

This matrix in turn allows us to define a covariant derivative $D$ defined for all tensor fields.

We will only need $D$ to differentiate functions and 1 -forms and content ourselves to give the definition for those cases.

If $f: M_{m} \rightarrow R$ is a real valued function then $D f=d f \in T^{*}(M)$, the cotangent bundle. The local coefficients $\left\{f_{i}\right\}$ defined by

$$
\left.d f\right|_{U}=\sum f_{i} \tau^{i}
$$

are called the first covariant derivatives relative to the local coframe.

If $w: M_{m} \rightarrow T^{*}(M)$ is a 1-form locally given by $\left.w\right|_{U}=\sum a_{i} \tau^{i}$, then $D$ is locally defined by $\left.D w\right|_{U}=\sum a_{i ; j} \tau^{i} \otimes \tau^{J} \in T^{*}(M) \otimes T^{*}(M)$ where the local coefficients $\left\{a_{i ; j}\right\}$ are defined by

$$
d a_{i}-\sum a_{j} \phi_{i}^{j}=\sum a_{i ; j} \tau^{j}
$$

and are called the second covariant derivatives relative to the local coframe.

We note that, in general, $a_{i ; j} \neq a_{j ; i}$, but in the special case $w=d f$ the differentiation of (1.1) implies that $f_{i ; j}=f_{j ; i}$.

An important reason for the introduction of the operator $D$ is the following formula for the derivative of the metric pairing $(w, \eta)$ between two 1-forms $w$ and $\eta$. If

$$
w=\sum a_{i} \tau^{i} \text { and } \eta=\sum b_{j} \tau^{j}
$$


then the skew-symmetry of the $\phi_{i}^{j}$ implies

$$
\begin{aligned}
d(w, \eta) & =d\left(\sum a_{i} b_{i}\right)=\sum d a_{i} b_{i}+a_{i} d b_{i} \\
& =\sum\left(d a_{i}-\sum a_{j} \phi_{i}^{j}\right) b_{i}+\sum a_{i}\left(d b_{i}-\sum b_{j} \phi_{i}^{j}\right) \\
& =(D w, \eta)+(w, d \eta) .
\end{aligned}
$$

We will write the length of a 1 -form $w$ by $\|w\|=(w, w)$ and note that as a consequence of the last equation $D w=0$ implies $\|w\|$-constant.

We define the Hessian of $f$ relative to $\mathrm{I}$ to be the symmetric quadratic differential form given by

$$
H_{\mathrm{I}}(f)=D(d f) .
$$

The metric trace of the Hessian defines a second order partial differential operator

$$
\Delta_{\mathrm{I}}(f)=\operatorname{Tr}_{\mathrm{I}} H_{\mathrm{I}}(f)=\sum f_{i ; i}
$$

called the Laplace operator of $\mathrm{I}$. The fundamental property of this operator is given by the Bochner lemma [30, p. 30].

LEMMA 1.1. Let $M_{m}$ be a compact Riemannian manifold without boundary. Then $\Delta_{\mathrm{I}}(f)=0$ implies $f=$ constant.

Now let $X: M_{m} \rightarrow R^{m+p}$ be an immersion. This induces a Riemannian metric $\mathrm{I}=d X \cdot d X$ called the induced metric, where

$$
\mathrm{I}([\alpha],[\beta])=\left.\left.\frac{d X \circ \alpha}{d t}\right|_{t=0} \cdot \frac{d X \circ \beta}{d t}\right|_{t=0}
$$

defines the bilinear pairing for tangent vectors with representative curves $\alpha$ and $\beta$.

Now if we view the frame bundle of $R^{m+p}$ as a group defined up to right translations, then the Mauer-Cartan equations induce equations called the structure equations of $\mathrm{E}$. Cartan. If $X$ is the position vector of $R^{m+p}$ and $\left(e_{1}, \ldots, e_{m+p}\right)$ is a local family of orthonormal frames, then the structure equations define 1-forms $\tau^{A}$ and $\phi_{A}^{B}$ via

$$
d X=\sum \tau^{A} e_{A} \text { and } d e_{A}=\sum \phi_{A}^{B} e_{B} .
$$

This implies $\phi_{A}^{B}=-\phi_{B}^{A}$ and the integrability conditions

$$
d \tau^{A}=\Sigma \tau^{B} \wedge \phi_{B}^{A} \quad \text { and } \quad d \phi_{A}^{B}=\Sigma \phi_{A}^{C} \wedge \phi_{C}^{B} .
$$

A good discussion of these equations may be found in [25, Vols. 2, 4].

By restricting $X$ to the position vector of an immersion $X: M_{m} \rightarrow R^{m+p}$ and $\left(e_{1}, \ldots, e_{m+p}\right)$ to local families of orthonormal frames with $e_{1}, \ldots, e_{m}$ tangent to $M_{m}$ we have 


$$
\tau^{m+1}=0, \ldots, \tau^{m+p}=0 .
$$

This implies that the induced metric is locally given by $I=\sum\left(\tau^{i}\right)^{2}$ and

$$
d \tau^{i}=\sum \tau^{j} \wedge \phi_{j}^{i} \quad \text { with } \phi_{i}^{j}=-\phi_{j}^{i},
$$

and hence that these 1-forms $\phi_{i}^{j}$ define the Levi-Civita connection matrix. The components $\phi_{i}^{j}=\sum \Gamma_{i k}^{j} \tau^{k}$ are known as the Christoffel symbols. The curvature matrix of this connection is defined by

$$
\Theta_{i}^{j}=d \phi_{i}^{j}-\Sigma \phi_{i}^{k} \wedge \phi_{k}^{j} .
$$

The components

$$
\Theta_{i}^{j}=\sum R_{i k l}^{j} \tau^{k} \wedge \tau^{l}
$$

define the Riemann curvature tensor.

The restrictions of the structure equations yield equations associated with the following classical names:

The Gauss equations:

$$
d \phi_{i}^{j}=\Sigma \phi_{i}^{k} \wedge \phi_{k}^{j}+\Sigma \phi_{i}^{a} \wedge \phi_{a}^{j}
$$

or equivalently, $\Theta_{i}^{j}=-\sum \phi_{i}^{a} \wedge \phi_{j}^{a}$;

The Codazzi-Mainardi equations:

$$
d \phi_{i}^{a}=\Sigma \phi_{i}^{j} \wedge \phi_{j}^{a}+\Sigma \phi_{i}^{b} \wedge \phi_{b}^{a}
$$

The Weingarten equations:

$$
d e_{a}=\sum \phi_{a}^{i} e_{i}+\sum \phi_{a}^{b} e_{b} .
$$

In addition the curvature matrix satisfies integrability conditions resulting from differentiation of the restrictions of the structure equations. These are:

First Bianchi identity:

$$
\sum \tau^{j} \wedge \Theta_{j}^{i}=0
$$

Second Bianchi identity:

$$
d \Theta_{i}^{j}+\Sigma \Theta_{i}^{k} \wedge \phi_{k}^{j}-\Sigma \phi_{i}^{k} \wedge \Theta_{k}^{j}=0 .
$$

In particular we have the following symmetry of the Riemann tensor:

$$
R_{i k l}^{j}=R_{k i j}^{l} \text {. }
$$

2. The vector valued second fundamental form. If we let $A \in R^{m+p}$, then the position vector of an immersion $X: M_{m} \rightarrow R^{m+p}$ allows us to define a real valued function by $X \cdot A$. This function is called the height function in the 
direction $A$. The Hessians of these functions characterize a normal vector valued quadratic differential form II via

$$
H_{\mathrm{I}}(X \cdot A)=\mathrm{II} \cdot A \text { for all } A \in R^{m+p} .
$$

In order to see that the range of II is normal vector valued we will explicitly compute the Hessian.

Since the structure equations give

$$
d(X \cdot A)=\sum e_{i} \cdot A \tau^{i}
$$

and

$$
d\left(e_{i} \cdot A\right)-\sum e_{j} \cdot A \phi_{i}^{j}=\sum e_{b} \cdot A \phi_{i}^{b},
$$

we are led to introduce coefficients

$$
\phi_{i}^{b}=\sum h_{i j}^{b} \tau^{j}
$$

where necessarily

$$
h_{i j}^{b}=h_{j i}^{b}
$$

since they are the coefficients of the Hessian of a function. Matters being so,

$$
\mathrm{II}=\sum \phi_{i}^{b} \otimes \tau^{i} \otimes e_{b}=\sum h_{i j}^{b} \tau^{i} \otimes \tau^{j} \otimes e_{b} \in T^{*} \otimes T^{*} \otimes N,
$$

which is a normal vector valued quadratic differential form.

Intuitively, II controls the convexity properties of the submanifold since it contains all information on the second derivatives of height functions. In order to see this more precisely we follow [13] and let $\alpha: R \rightarrow M$ be a curve parameterized by arc length $\sigma$ and having tangent vector $T$ on $M$. If we choose local orthonormal frames with $e_{1}=T$ and compute the first curvature $\kappa$ and the first normal $N$ of $X \circ \alpha: R \rightarrow R^{m+p}$, then

$$
\begin{aligned}
\kappa N & =d^{2} / d \sigma^{2}(X \circ \alpha)=\left\langle e_{1}, d e_{1}\right\rangle \\
& =\left\langle e_{1}, \sum \phi_{1}^{j} e_{j}+\sum \phi_{1}^{a} e_{a}\right\rangle=\sum \Gamma_{11}^{j} e_{j}+\sum h_{11}^{a} e_{a} .
\end{aligned}
$$

The term $\sum \Gamma_{11}^{j} e_{j}=0$ if and only if the curve $\alpha$ is a geodesic, in which case we have the simple geometric statement

$$
\kappa N=\mathrm{II}\left(e_{1}, e_{1}\right) \text {. }
$$

We remark that those directions $z$ with II $(z, z)=0$ are called asymptotic directions.

A basic pointwise algebraic invariant of $\mathrm{II}$ is the rank $r$ defined for $u \in M$ by

$$
r(u)=m+p-\operatorname{dim}\{A \mid \mathrm{II}(u) \cdot A=0\}
$$


Now recalling that the first osculating space of $M$ at $u$, denoted by $O_{u}^{1}$, is the vector space generated by all first normals to curves lying in $M$, and that every unit vector tangent to $M$ at $u$ can be realized as the tangent vector to a geodesic, we see that (2.1) implies $\operatorname{dim} O_{u}^{1}=m+r(u)$.

One of the simplest geometric properties that II detects is whether the immersion is substantial [19, p. 212], that is, the image $X\left(M_{m}\right)$ does not lie in a subplane of $R^{m+p}$.

Proposition 2.1. Let $X: M_{m} \rightarrow R^{m+p}$ be an immersion and let $A \in R^{m+p}$ be normal at a point $X(u)$. Then $X\left(M_{m}\right)$ lies in a hyperplane perpendicular to $A$ if and only if II $\cdot A=0$.

Proof. If $X\left(M_{m}\right)$ lies in a hyperplane perpendicular to $A$, then $A$ is perpendicular to every osculating space and hence II $\cdot A=0$. Conversely if

$$
0=\mathrm{II} \cdot A=H_{\mathrm{I}}(X \cdot A)=D(d(X \cdot A)),
$$

then equation (1.2) implies $\|d(X \cdot A)\|=$ constant. Now since $A$ is normal at $u$,

$$
d(X \cdot A)=\sum e_{i} \cdot A \tau^{i}=0
$$

at $u$, and hence $d(X \cdot A)=0$ identically. As such we have $X \cdot A=$ constant, which implies that $X\left(M_{m}\right)$ lies in a hyperplane with normal direction parallel to $A$.

The condition that there exists a point $u$ such that $A$ is normal at $X(u)$ is necessary, since $A$ might be a direction which is always tangent. This would happen for example if a translate of $A$ were contained in the image $X\left(M_{m}\right)$. This condition is automatically satisfied, however, if $M_{m}$ is compact without boundary. In order to see this let $A \in R^{m+p}$; then there is at least one point where the height function $X \cdot A$ has a critical point. At such a point $0=d(X \cdot A)=\sum e_{i} \cdot A \tau^{i}$ and hence $A$ is normal.

We may globalize the notion of osculating space to get the minimal enveloping space $\mathscr{E}\left(M_{m}\right)$, which is the linear subspace of $R^{m+p}$ generated as the union of the translates to the origin of all the first osculating spaces. Thus by abuse of notation

$$
\mathscr{E}\left(M_{m}\right)=\cup_{u} O_{u}^{1} .
$$

This terminology is justified since $A$ is perpendicular to $\mathscr{E}\left(M_{m}\right)$ if and only if $A$ is everywhere normal to $X\left(M_{m}\right)$ and II $\cdot A=0$. Hence the last Proposition implies that $X\left(M_{m}\right)$ is contained substantially in a translate of $\mathscr{E}\left(M_{m}\right)$.

In case the manifold $M_{m}$ is compact without boundary, it is much easier to characterize substantial immersions.

The basic invariant needed is the metric trace of II, which is the normal vector field

$$
H=\operatorname{tr}_{\mathrm{I}} \mathrm{II}=\sum h_{i i}^{a} e_{a}
$$

called the mean curvature field. 
Since II $\cdot A=H_{\mathrm{I}}(X \cdot A)$ we have the analytical interpretation of the mean curvature field that

$$
H \cdot A=\Delta_{\mathrm{I}}(X \cdot A) .
$$

In particular the Bochner Lemma immediately implies the following proposition.

Proposition 2.2. Let $X: M_{m} \rightarrow R^{m+p}$ be an immersion of a compact $M_{m}$ without boundary and let $A \in R^{m+p}$. Then $X\left(M_{m}\right)$ lies in a hyperplane perpendicular to $A$ if and only if $H \cdot A=0$.

As a result $\mathscr{E}\left(M_{m}\right)=\{A \mid H \cdot A=0\}$ for $M_{m}$ compact without boundary.

We will not give a systematic report on the properties and applications of $H$. The interested reader is referred to [9].

3. Subspaces of tensor and exterior products. Let $V$ be an $m$-dimensional and $W$ an $n$-dimensional vector space over a field $K$, and let $\mathcal{C} \subset V \otimes_{K} W$ be an $r$ dimensional subspace. The subspace $\mathcal{L}$ defines unique subspaces $V^{\mathfrak{L}}$ of $V$ and $W^{\mathfrak{L}}$ of $W$, called the minimal enveloping subspaces of $\mathcal{L}$, which are characterized by their minimality with respect to the inclusions $\mathcal{L} \subseteq V^{\mathfrak{L}} \otimes W^{\mathfrak{L}} \subseteq V \otimes W$.

The existence and uniqueness of these minimal enveloping subspaces will be systematically exploited in the algebraic analysis of the vector valued second fundamental form and in the study of the Gauss equations. As such this section is devoted to a short report on the foundations of this concept.

Let $v^{*} \in V^{*}$ be a linear functional on $V$. Then $v^{*}$ may be extended to a linear map

$$
\left.v^{*}\right\lrcorner: V \otimes W \rightarrow W
$$

by covering the bilinear map

$$
v^{*}(v, w)=v^{*}(v) w, \quad v \in V, w \in W .
$$

Analogously $w^{*} \in W$ may be extended to a linear map $\left.w^{*}\right\lrcorner: V \otimes W \rightarrow V$.

Proposition 3.1. The minimal enveloping subspaces of $\mathcal{L} \subseteq V \otimes W$ are realized by

$$
\left.\left.V^{\mathfrak{L}}=\left\{w^{*}\right\lrcorner \mathfrak{L} \mid w^{*} \in W^{*}\right\} \text { and } W^{\mathfrak{L}}=\left\{v^{*}\right\lrcorner \mathfrak{L} \mid v^{*} \in V^{*}\right\} .
$$

Proof. Let $\left\{f_{a}\right\}$ be a basis of $V,\left\{e_{\alpha}\right\}$ a basis of $W$ and $\left\{1_{A}\right\}$ a basis for $\mathcal{L}$ Then writing $1_{A}=\sum v_{A}^{\alpha} \otimes e_{\alpha}=\sum f_{a} \otimes w_{A}^{a}$ we see that the above definitions become

$$
V^{\mathfrak{L}}=\left\{v_{A}^{\alpha}\right\} \quad \text { and } \quad W^{\mathfrak{L}}=\left\{w_{A}^{a}\right\} .
$$

If $\mathscr{L} \subseteq V^{\mathfrak{L}} \otimes W^{\mathfrak{L}}$ as described here, then the factors are certainly minimal. In order to see the inclusion we choose the above bases so that $f_{1}, \ldots, f_{s}$ is a 
basis of $V^{\mathfrak{L}}$ and $e_{1}, \ldots, e_{r}$ is a basis of $W^{\mathfrak{L}}$. Now let $\left\{f_{a}^{*}\right\}$ be the dual basis of $V^{*}$ and $\left\{e_{\alpha}^{*}\right\}$ be the dual basis of $W^{*}$.

Since the canonical bilinear pairing

$$
\left\langle f_{a}^{*} \otimes e_{\alpha}^{*}, \mathfrak{L}\right\rangle=0
$$

for $(s+1 \leqslant a \leqslant m, 1 \leqslant \alpha \leqslant n)$ and for $(1 \leqslant a \leqslant m, r+1 \leqslant \alpha \leqslant n)$ we see that

$$
\mathfrak{L} \subseteq\left(\left\{V^{\mathfrak{L}} \otimes W^{\mathfrak{L}}\right\}^{\perp}\right)^{\perp}=V^{\mathfrak{L}} \otimes W^{\mathfrak{L}} .
$$

Using duality we can give a second description of the minimal enveloping subspaces by

$$
\left.\left.V^{\mathfrak{L}}=\left\{v^{*} \in V^{*} \mid v^{*}\right\lrcorner \mathfrak{L}=0\right\}^{\perp} \text { and } W^{\mathfrak{L}}=\left\{w^{*} \in W^{*} \mid w^{*}\right\lrcorner \mathfrak{L}=0\right\}^{\perp} .
$$

Now let $T \in V \otimes W$ and let $\mathcal{L}$ be the 1-dimensional vector space generated by $T$. If we write $T$ as a minimal length sum of monomials

$$
T=\sum v_{i} \otimes w_{i}, \quad v_{i} \in V, w_{i} \in W,
$$

then necessarily $\left\{v_{i}\right\}$ are linearly independent and $\left\{w_{i}\right\}$ are linearly independent. Hence

$$
\operatorname{dim} V^{\mathfrak{L}}=\operatorname{dim}\left\{v_{i}\right\}=\operatorname{dim}\left\{\boldsymbol{w}_{i}\right\}=\operatorname{dim} W^{\mathfrak{L}} .
$$

This common integer is called the rank of the tensor $T$ [7].

A refinement of these notions arises when a subspace $\mathcal{L}$ is contained in an exterior power of a vector space $\mathcal{L} \subseteq \Lambda^{p} V$.

Again the subspace $\mathfrak{L}$ defines a unique minimal enveloping subspace $V^{\mathfrak{L}}$ of $V$ characterized by minimality with respect to the inclusions

$$
\mathfrak{L} \subseteq \Lambda^{p} V^{\mathfrak{L}} \subseteq \Lambda^{p} V .
$$

If $v^{*}$ is a linear functional on $V$, then $v^{*}$ may be extended to a unique linear mapping

$$
\left.\nu^{*}\right\lrcorner: \Lambda^{p} V \rightarrow \Lambda^{p-1} V,
$$

characterized on monomials by the antiderivation rule

$$
\begin{aligned}
& \left.v^{*}\right\lrcorner\left(x_{1} \wedge \cdots \wedge x_{p}\right) \\
& =\sum_{j=1}^{p}(-1)^{j-1}\left\langle x_{j}, v^{*}\right\rangle x_{1} \wedge \cdots \wedge x_{j-1} \wedge x_{j+1} \wedge \cdots \wedge x_{p} .
\end{aligned}
$$

Now by using the duality theory of exterior algebras or by applying a general result $\left[26\right.$, p. 29] one easily sees that $V^{\mathfrak{L}}$ has the simple description

$$
\left.V^{\mathfrak{L}}=\left\{v^{*} \in V^{*} \mid v^{*}\right\lrcorner \mathfrak{L}=0\right\}^{\perp} \text {. }
$$


The principal application of these ideas will be to the analysis of bilinear mappings $b: V \times V \rightarrow W$. Such a mapping defines an element

$$
b \in V^{*} \otimes V^{*} \otimes W,
$$

which may be assigned two different tensor ranks.

The first rank which we write $\operatorname{rank}_{W} b$ and call the target rank, is obtained by viewing $b \in\left(V^{*} \otimes V^{*}\right) \otimes W$.

The second rank, which we write $\operatorname{rank}_{V} b$ and call the source rank, is obtained by viewing $b \in V^{*} \otimes\left(V^{*} \otimes W\right)$.

A bilinear mapping $b: V \times V \rightarrow W$ is said to have no left conjugate space if

$$
b(u, v)=0 \quad \text { for all } v \in V \text { implies } \mathrm{u}=0 .
$$

If we introduce the linear system

$$
l=\{b(\cdot, v) \mid v \in V\} \subseteq\left(V^{*} \otimes W\right)^{b} \subseteq V^{*} \otimes W,
$$

then statement (3.1) holds if and only if $u\lrcorner l=0$ implies $u=0$, and this holds if and only if $u\lrcorner l=0$ implies $u\lrcorner V^{* l}=0$. Hence $b$ has no left conjugate space if and only if $V^{* l}=V^{*}$. A symmetric bilinear map with no left and hence no right conjugate space is called essential.

4. Invariants of the second fundamental form. We have seen that the second fundamental form II is a normal vector valued quadratic differential form and hence gives rise to symmetric bilinear mappings

$$
\text { II }(u): T_{u} \times T_{u} \rightarrow N_{u} .
$$

Applying the results of the last section we see that II $(u)$ has two tensor ranks and several associated enveloping spaces.

The target rank $\operatorname{dim}_{N}$ II is obtained by viewing

$$
\text { II }(u)=\Sigma \tau^{i} \otimes \tau^{j} \otimes h_{i j}^{b} e_{b} \in\left(T^{*} \otimes T^{*}\right) \otimes N,
$$

and hence

$$
\begin{aligned}
\operatorname{dim}_{N} \text { II }(u) & =\operatorname{dim}\left\{\sum h_{i j}^{b} e_{b}\right\} \\
& =m+p-\operatorname{dim}\{A \mid \mathrm{II}(u) \cdot A=0\}=r(u) .
\end{aligned}
$$

Thus the target rank is the integer we previously called the rank of II.

The source rank $\operatorname{dim}_{T}$ II is obtained by viewing

$$
\text { II }(u)=\sum \phi_{i}^{b} \otimes \tau^{i} \otimes e_{b} \in T^{*} \otimes\left(T^{*} \otimes N\right),
$$

and hence,

$$
\operatorname{dim}_{T} \text { II }(u)=\operatorname{dim}\left\{\phi_{i}^{b}\right\} .
$$

We define the dual dimension of $M$ at a point $u$ to be the source $\operatorname{rank} \operatorname{dim}\left\{\phi_{i}^{b}\right\}$, 
and will denote this integer by $s(u)$. The reason for this nomenclature will be exhibited in the next section where we give $s(u)$ a geometric interpretation. The complementary dimension $m-s$ is known as the index of relative nullity [13, p. 425].

If we view II $\in\left(T^{*} \otimes T^{*}\right) \otimes N$, then there are two minimal enveloping subspaces, characterized by their minimality with respect to the inclusion II $\in\left(T^{*} \otimes T^{*}\right)^{\mathrm{II}} \otimes N^{\mathrm{II}}$. In order to save writing we introduce the notation

$$
\mathcal{L}=\left(T^{*} \otimes T^{*}\right)^{\mathrm{II}}
$$

which is the linear system of quadratic forms

$$
\mathcal{L}=\left\{\sum \lambda_{b} \mathrm{II}^{b}\right\} \text { for } \lambda_{b} \in R \text {. }
$$

On the other hand if we view II $\in T^{*} \otimes\left(T^{*} \otimes N\right)$, then there are two more minimal enveloping subspaces characterized by their minimality with respect to the inclusion II $\in T^{* \text { II }} \otimes\left(T^{*} \otimes N\right)^{\mathrm{II}}$. Again to save writing we introduce the notation

$$
\Phi=\left(T^{*} \otimes N\right)^{\mathrm{II}},
$$

which is the linear system of tensors

$$
\Phi=\left\{\sum \mu_{i} \phi_{i}^{b} \otimes e_{b}\right\}, \quad \mu_{i} \in R .
$$

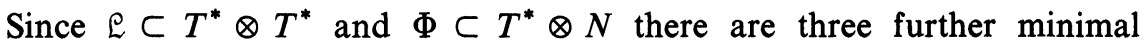
enveloping subspaces, characterized by their minimality with respect to the inclusions $\mathcal{L} \subset T^{* \mathcal{L}} \otimes T^{* \mathcal{L}}$ and $\Phi \subset T^{* \Phi} \otimes N^{\Phi}$. The collection of minimal enveloping subspaces we have introduced is not disjoint; in fact,

$$
\left\{\phi_{i}^{a}\right\}=T^{* \Phi}=T^{* \mathrm{II}}=T^{* \complement} \text { and }\left\{\sum h_{i j}^{b} e_{b}\right\}=N^{\mathrm{II}}=N^{\Phi} .
$$

Matters being so, we have the following basic invariants of II:

(A) Two tensor ranks:

(1) the rank $r$,

(2) the dual dimension $s$.

(B) Two linear systems of tensors:

(1) $\mathcal{E}=\left\{\sum \lambda_{b} \mathrm{II}^{b}\right\} \subset T^{*} \otimes T^{*}$,

(2) $\Phi=\left\{\sum \mu_{i} \phi_{i}^{b} \otimes e_{b}\right\} \subset T^{*} \otimes N$.

(C) Two linear subspaces:

(1) $T^{* \mathrm{II}}=\left\{\phi_{i}^{a}\right\}$

(2) $N^{\mathrm{II}}=\left\{\sum h_{i j}^{b} e_{b}\right\}$.

We begin with normalizations related to $\mathcal{L}$ and $N^{\mathrm{II}}$. Let us agree from now on to choose an orthonormal basis for $N$ adapted to $N^{\text {II }}$. Thus

$$
N^{\mathrm{II}}=\left\{e_{m+1}, \ldots, e_{m+r}\right\} \text {. }
$$

This implies 


$$
h_{i j}^{a}=0 \quad(m+r+1 \leqslant a \leqslant m+p)
$$

and hence

$$
\phi_{i}^{a}=0 \quad \text { and } \quad \mathrm{II}^{a}=0 \quad(m+r+1 \leqslant a \leqslant m+p) .
$$

Next we define a symmetric bilinear form $(,)_{N}: N \times N \rightarrow R$ by

$$
(\xi, \eta)_{N}=\operatorname{trace}\left(\mathrm{I}^{-1} \xi \cdot \mathrm{II}\right)\left(\mathrm{I}^{-1} \eta \cdot \mathrm{II}\right)
$$

where $I$ is the metric tensor. In orthonormal frames

$$
(\xi, \eta)_{N}=\sum \xi \cdot e_{a} \eta \cdot e_{b} h_{i j}^{a} h_{i j}^{b} .
$$

The matrix of $(,)_{N}$ in such a basis is

$$
S_{a b}=\left(e_{a}, e_{b}\right)_{N}=\sum h_{i j}^{a} h_{i j}^{b} .
$$

If we diagonalize $(,)_{N}$ by an orthogonal change of base then

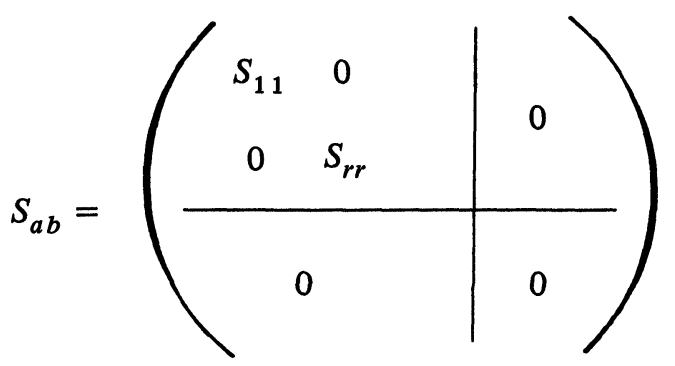

with the diagonal terms $S_{a a}=\Sigma\left(h_{i j}^{a}\right)^{2}>0$.

As a result we see that $\operatorname{rank}(,)_{N}=\operatorname{dim} \mathcal{L}=r$, and that the restriction $(,)_{N}: N^{\text {II }} \times N^{\text {II }} \rightarrow R$ is positive definite. This last remark is a potentially powerful statement. If, for example, $p=2$ and

$$
\mathrm{II}^{m+1}=\operatorname{diag}\left(a_{1}, \ldots, a_{m}\right) \text { and } \mathrm{II}^{m+2}=\operatorname{diag}\left(b_{1}, \ldots, b_{m}\right)
$$

then

and the positivity of this matrix implies

$$
S_{a b}=\left(\begin{array}{cc}
\sum a_{i}^{2} & \sum a_{i} b_{i} \\
\sum a_{i} b_{i} & \sum b_{i}^{2}
\end{array}\right)
$$

$$
0 \leqslant \operatorname{det} S_{a b}=\Sigma a_{i}^{2} \Sigma b_{i}^{2}-\left(\sum a_{i} b_{i}\right)^{2}
$$

which is the Cauchy-Schwarz inequality. 
There is an obvious globalization of this semidefinite form to global normal vector fields by integration:

$$
(\xi, \eta)_{g}=\int_{M}(\xi, \eta)_{N} d A \text { where } \int_{M} d A=1
$$

This has not been well investigated, but may be useful in the study of extrinsic problems. An example of such a result is

THEOREM 4.1. Let $X: M_{m} \rightarrow R^{m+p}$ be an isometric immersion of a compact Riemannian manifold without boundary. If $X_{N}=$ normal projection of $X$ then $\left(X_{N}, X_{N}\right)_{g} \geqslant m$ with equality if and only if the immersion lies on a sphere centered at the origin.

Proof. Consider the energy function $E=X \cdot X / 2$. Since

$$
d E=X \cdot d X=\sum X \cdot e_{i} \tau^{i}
$$

the Hessian is easily computed to be

$$
H_{\mathrm{I}}(E)=\sum \delta_{i j}+\sum X \cdot e_{a} h_{i j}^{a} \tau^{i} \otimes \tau^{j}=\mathrm{I}+X \cdot \mathrm{II}
$$

Now let $F=H_{\mathrm{I}}(E)$; then

$$
0 \leqslant \int_{M} \operatorname{trace} F^{t} F d A=\int_{M} m+2 X \cdot H+\operatorname{trace} X, \text { II } X \cdot \text { II } d A,
$$

and Minkowski's formula

$$
0=\int_{M} \Delta_{\mathrm{I}} E d A=\int_{M} m+X \cdot H d A
$$

yields the inequality. The equality forces $F=0$ and as a result

$$
0=\operatorname{trace} F=\Delta_{\mathrm{I}} E,
$$

and the Bochner lemma may be applied to give $E=X \cdot X / 2=$ constant.

Since several arguments to come are based on adapting orthonormal frames to a subspace of the linear system $\Phi$, we will discuss this procedure in a unified way. First we note the dimension of the linear system $\Phi$ is computable by inspection and has value $\operatorname{dim} \Phi=s$.

Proposition 4.2. Let $\Psi_{1}$ be a subspace of $\Phi$ with basis $\left\{\phi^{1}, \ldots, \phi^{j}\right\}$. Then there exists a choice of orthonormal basis such that $\Psi_{1}$ has basis $\sum \phi_{1}^{a} \otimes e_{a}, \ldots$, $\sum \phi_{j}^{a} \otimes e_{a}$.

Proof. Let $\phi^{\lambda}=\sum \mu_{\lambda i} \phi_{i}^{a} \otimes e_{a}$, and introduce $x_{\lambda}=\sum \mu_{\lambda i} e_{i}$. These vectors $x_{1}, \ldots, x_{j}$ are linearly independent since a relation $0=\sum b_{\lambda} x_{\lambda}=\sum b_{\lambda} \mu_{\lambda i} e_{i}$ implies $\sum b_{\lambda} \phi^{\lambda}=\sum b_{\lambda} \mu_{\lambda i} \phi_{i}^{a}=0$, and hence that $b_{\lambda}=0$.

Now choose $e_{1}, \ldots, e_{j}$ to be an orthonormal base for the subspace $x_{1}, \ldots, x_{j}$. As such $e_{\mu}=H_{\mu \lambda} x_{\lambda}$. Now since $\phi^{\lambda}=\mathrm{II}\left(x_{\lambda},\right)$, we see that 


$$
H_{\mu \lambda} \phi^{\lambda}=\mathrm{II}\left(H_{\mu \lambda} x_{\lambda},\right)=\mathrm{II}\left(e_{\mu},\right)=\sum \phi_{\mu}^{a} \otimes e_{a} .
$$

Since $H_{\mu \lambda}$ is nonsingular, $\Psi_{1}$ has the new basis $\sum \phi_{1}^{a} \otimes e_{a}, \ldots, \Sigma \phi_{j}^{a} \otimes e_{a}$.

If we are free to choose the complement of $\Psi_{1}$ in $\Phi$ then we can arrange $\Phi=\Psi_{1}+\Psi_{2}$ with basis $\psi_{1}=\left\{\Sigma \phi_{\mu}^{a} \otimes e_{a}\right\}$ and $\Psi_{2}=\left\{\Sigma \phi_{\sigma}^{a} \otimes e_{a}\right\}$ where $1 \leqslant \mu \leqslant j$ and $j+1 \leqslant \sigma \leqslant s$.

Explicit choices of basis for $\Phi$ will depend on properties of the curvature matrix and will be discussed in the section entitled the Gauss sequence.

As a result of the discussion on the conjugate space of symmetric bilinear maps in $\$ 3$ we have the following result.

Proposition 4.3. The second fundamental form II is essential at a point $u \in M$, i.e. II $(x, z)=0$ for all $x \in T_{u}$ implies $z=0$, if and only if $s=m$.

Proof. The bilinear map II is essential if and only if $T^{* I I}=T^{*}$ and this happens if and only if $s=m$.

An important result is that compact manifolds without boundary always have a neighborhood on which the second fundamental form is essential.

Proposition 4.4. Let $X: M_{m} \rightarrow R^{m+p}$ be an isometric immersion of a compact Riemannian manifold without boundary. Then there exists a point $u \in M$ such that $s(u)=m$.

Proof. Again we consider the energy function $E=X \cdot X / 2$. By compactness $E$ has a maximum at some point $u \in M_{m}$. We have seen in Theorem 4.1 that the Hessian of $E$ is given by $H_{\mathrm{I}}(E)=\mathrm{I}+X \cdot \mathrm{II}$ and this is negative definite at $u$ since $u$ is a maximum. Now $I$ is positive definite which means that $X \cdot$ II is certainly negative definite. Since a component of II is negative definite we have immediately that $\mathrm{II}(u)$ is essential and hence by the last proposition that $s(u)=m$.

Next we give a short report on the construction of functions from the second fundamental form. In the classical case of a hypersurface where $X: M_{m} \rightarrow R^{m+1}$, a choice of normal determines a matrix $\mathrm{II}^{m+1}$ via II $=\mathrm{II}^{m+1} e_{m+1}$ and hence a hypersurface determines a matrix $\mathrm{II}^{m+1}$ up to sign. In this case

$$
\operatorname{det}\left(\mathrm{I}+\lambda \mathrm{II}^{m+1}\right)=\Sigma\left(\begin{array}{c}
m \\
i
\end{array}\right) \sigma_{i} \lambda^{i}
$$

defines $m$ functions $\sigma_{i}$ with the even functions uniquely defined and the odd functions defined up to sign.

The calculation

$$
\left(\tau^{1}+\lambda \phi_{1}^{m+1}\right) \wedge \cdots \wedge\left(\tau^{m}+\lambda \phi_{m}^{m+1}\right)=\left(\Sigma\left(\begin{array}{c}
m \\
i
\end{array}\right) \sigma_{i} \lambda^{i}\right) \tau^{1} \wedge \cdots \wedge \tau^{m}
$$

and the Gauss equations

$$
\Theta_{i}^{j}=-\phi_{i}^{m+1} \wedge \phi_{j}^{m+1}
$$


exhibit that all the even functions $\sigma_{2 i}$ are in fact intrinsic invariants resulting from the sums of wedge products of the coframe basis and elements of the curvature matrix. Moreover, these are the point averages of the $2 i$ th sectional curvatures.

Generalizing the hypersurface idea we may expand $\operatorname{det}\left(\mathrm{I}+\sum \lambda_{a} \mathrm{II}^{a}\right)$ in powers of the unknowns $\lambda_{1}, \ldots, \lambda_{r}$. Thus, as above,

$$
\begin{aligned}
\left(\tau^{1}+\sum \lambda_{a} \phi_{1}^{a}\right) \wedge \cdots \wedge & \left(\tau^{m}+\sum \lambda_{a} \phi_{m}^{a}\right) \\
=\left(1+\sum T^{a_{1}} \lambda_{a_{1}}\right. & +\sum T^{a_{1} a_{2}} \lambda_{a_{1}} \lambda_{a_{2}}+\cdots \\
& \left.+\sum T^{a_{1}} \cdots a_{r} \lambda_{a_{1}} \cdots \lambda_{a_{r}}\right) \tau^{1} \wedge \cdots \wedge \tau^{m},
\end{aligned}
$$

where now the terms $T^{a_{1} \cdots a_{j}}$ define polynomials of degree $j$ on the normal bundle. By direct calculation one may show that $T^{a_{1}}=H^{a_{1}}$ are the components of the mean curvature vector and $T^{a_{1} a_{2}}=H^{a_{1}} H^{a_{2}}-S_{a_{1} a_{2}}$ where $S_{a_{1} a_{2}}$ is the matrix associated to the bilinear form $(,)_{N}$.

We may construct scalar functions on $M_{m}$ from these polynomials on the normal bundle by invariant theoretical devices. For example from the components $T^{a_{1}}$ we have

$$
H \cdot H=\text { length of mean curvature vector }
$$

and from the components $T^{a_{1} a_{2}}$ we may construct a sequence of invariants $\eta_{1}, \ldots, \eta_{r}$ by

$$
\operatorname{det}\left(\mathrm{I}_{a b}+\lambda T^{a b}\right)=\sum_{c=1}^{r}\left(\begin{array}{l}
r \\
c
\end{array}\right) \eta_{c} \lambda^{c}
$$

The simplest of this last sequence is the function

$$
\eta_{1}=\operatorname{trace} T^{a b}=\sum H^{a} H^{a}-\operatorname{trace} \mathrm{II}^{a} \mathrm{II}^{a}=H \cdot H-N(\mathrm{II}) .
$$

Using the Gauss equations $\eta_{1}$ may be shown to be a multiple of the scalar curvature $R=1 / m(m-1) \sum R_{i i j}^{j}$. The other functions $\eta_{2}, \ldots, \eta_{r}$ to my knowledge have not been investigated. Since they are not particularly complex, an analysis of these functions, hopefully with global integral formulas, would seem a fruitful line of research.

There are, of course, invariants of the higher order terms, but with the exclusion of two special constructions which appear in the next section, this is unexplored territory.

5. Tubes and Gauss maps. Let $X: M_{m} \rightarrow R^{m+p}$ be an isometric immersion of a Riemannian manifold $M_{m}$. Then a natural mapping $g: M_{m} \rightarrow G(m, m+p)$ into the Grassmannian of $m$-planes in $(m+p)$-space is obtained by defining $g(u)$ to be the translate of the tangent $m$-plane at $X(u)$ to the origin.

If we view $G(m, m+p) \subset \Lambda^{m} R^{m+p}$ via the standard Plucker imbedding [25, p. 16] then $g(u)=e_{1} \wedge \cdots \wedge e_{m}$ may be interpreted as the position vector of a mapping $g: M_{m} \rightarrow \Lambda^{m} R^{m+p}$. 
Proposition 5.1. Let $X: M_{m} \rightarrow R^{m+p}$ be an isometric immersion of a Riemannian manifold $M_{m}$. Then the rank of the Gauss mapping $g$ at $u$ equals the dual dimension $s(u)$.

Proof. Since

$$
d\left(e_{1} \wedge \cdots \wedge e_{m}\right)=\sum \phi_{\alpha}^{a} e_{1} \wedge \cdots \wedge e_{\alpha-1} \wedge e_{a} \wedge e_{\alpha+1} \wedge \cdots \wedge e_{m},
$$

and the $e_{1} \wedge \cdots \wedge e_{\alpha-1} \wedge e_{a} \wedge e_{\alpha+1} \wedge \cdots \wedge e_{m}$ are orthonormal unit vectors in $\Lambda^{m} R^{m+p}$, we see that

$$
\operatorname{rank} g_{*}=\operatorname{dim}\left\{\phi_{\alpha}^{a}\right\}=s(u) .
$$

Thus the dual dimension geometrically represents the number of parameters upon which the tangent planes locally depend.

There is a second Gauss mapping which is defined from the unit normal bundle $N_{0}(M), G: N_{0}\left(M_{m}\right) \rightarrow S^{m+p-1}$, by assigning to $\nu \in N_{0}(M)$ its translate to the origin on the unit sphere. This last mapping has a local degree called the Lipschitz-Killing curvature. The local degree equals det $\nu \cdot$ II and is characterized by

$$
\underbrace{\left.G^{*} d \Sigma_{m+p-1}\right|_{v}}_{\text {area } S^{m+p-1}}=\operatorname{det} \nu \cdot \text { II } \underbrace{d \Sigma_{p-1}}_{\text {area fiber area base }} \underbrace{d A}
$$

The integral over the fiber of the absolute value

$$
\kappa(u)=\int|\operatorname{det} \nu \cdot \mathrm{II}| d \Sigma_{p-1}
$$

defines a function called the total curvature at $U$.

The rich algebraic geometric theory of the Gauss mapping

$$
g: M_{m} \rightarrow G(m, m+p)
$$

and the Chern-Lashof theory of the total absolute curvature have been discussed in Chern [10] and Gardner [15]. Hence we will not report further on these ideas here.

A possible interaction between these two Gauss maps could result from the analysis of the diagram

$$
N_{0}(M) \underset{G}{\longrightarrow} S^{m+p-1} \searrow_{G(m, m+p) \underset{g}{\longleftarrow} M}^{O(m+p)}
$$

where $O(m+p)$ is the group of orthogonal $(m+p) \times(m+p)$ matrices. This is the classical data for integral geometric investigation. The idea is that information on the left and right may be compared by lifting and integrating over fibers. This gives rise to functional transforms and to an incidence 
function. This would be fruitful if simple geometric phenomena on one side are translated into more complicated geometric phenomena on the other side.

The idea of integrating over the fiber of the normal bundle can be applied to the polynomials on the normal bundle defined in the last section. A convenient way to represent the terms which are homogeneous of degree $j$ is via the equations

$$
\operatorname{det}\left(I+\mu \sum \lambda_{a} \mathrm{II}^{a}\right)=1+\psi_{1} \mu+\cdots+\psi_{m} \mu^{m} .
$$

In [29] $\mathrm{H}$. Weyl showed by an elegant argument using classical invariant theory that the functions

$$
H_{j}(u)=\int \psi_{j}\left(\lambda_{m+1}, \ldots, \lambda_{m+p}\right) d \Sigma_{p-1}
$$

which are now defined on $M_{m}$ are actually intrinsic. In fact they are the averages of the $p$ th sectional curvatures introduced by Allendoerfer [1]. These particular functions arise in the study of $V\left(T_{\rho}\right)$, the volume of the normal tube of radius $\rho$ about the submanifold.

An easy calculation [29] shows

$$
\begin{aligned}
V\left(T_{\rho}\right)= & \int \tau^{1} \wedge \cdots \wedge \tau^{m} \\
& \times \int_{\Sigma \lambda_{a}^{2} \leqslant \rho} \operatorname{det}\left(\delta_{i j}+\sum \lambda_{a} h_{i j}^{a}\right) d \lambda_{m+1} \wedge \cdots \wedge d \lambda_{m+p} \\
= & \int d A \int_{\Sigma \lambda_{a}^{2} \leqslant \rho}\left(1+\psi_{1} \mu+\cdots+\psi_{m} \mu^{m}\right) d \lambda_{m+1} \wedge \cdots \wedge d \lambda_{m+p} \\
= & \int \Sigma c_{j} H_{j} d A \rho^{m+j}
\end{aligned}
$$

where the $c_{j}$ are universal constants.

Since these intrinsic functions $H_{j}$ appear so naturally in the deformation of submanifolds they are obvious candidates for integral formula analysis and, as a result, global theorems. As far as we know such theorems are not yet known.

6. Invariants of the curvature matrix. The curvature matrix $\left(\Theta_{i}^{j}\right)$ gives rise to a linear system

$$
\Theta=\left\{\Sigma a_{i j} \Theta_{i}^{j}\right\} \subset \Lambda^{2} T^{*}, \quad a_{i j} \in R,
$$

which has the minimal enveloping subspace

$$
\left.T^{* \Theta}=\{x \in T \mid x\lrcorner \Theta_{i}^{j}=0\right\}^{\perp} .
$$

The curvature rank of $M$ at a point $u$ is defined to be the dimension of $T^{* \Theta}$ at $u$, and will be denoted by $h(u)$. The complementary dimension $m-h$ is known as the index of nullity [13, p. 425].

The nomenclature for $h$ was chosen because of the next algebraic result characterizing $h$ as the rank of the curvature matrix in the usual sense of block diagonal reduction. 
Proposition 6.1. The integer $h$ is characterized as the smallest integer such that there exists a basis of $T^{*}$ with

$$
\Theta_{i}^{j}=\left(\begin{array}{c|c}
\Theta_{\alpha}^{\beta} & 0 \\
\hline 0 & 0
\end{array}\right) \quad(1 \leqslant \alpha, \beta \leqslant h) .
$$

Proof. Let $\tau^{1}, \ldots, \tau^{h}$ be an orthonormal basis for $T^{* \Theta}$. By definition $\Theta_{i}^{j} \in \Lambda^{2} T^{* \Theta}$, and hence the first Bianchi identity, $0=\sum \tau^{i} \wedge \Theta_{i}^{j}$, implies

$$
\Theta_{\lambda}^{j}=0 \quad(h+1 \leqslant \lambda \leqslant m) .
$$

Since $\Theta_{i}^{j}$ is skew-symmetric this gives the desired block diagonal form.

Conversely let us assume $\Theta_{\lambda}^{j}=0(h+1 \leqslant \lambda \leqslant m)$. The standard symmetry on pairs of indices of the curvature tensor then gives

$$
0=R_{\lambda k l}^{j}=R_{k \lambda j}^{l} \quad(h+1 \leqslant \lambda \leqslant m),
$$

which implies $\Theta_{i}^{j} \in \Lambda^{2}\left(\tau^{1}, \ldots, \tau^{h}\right)$ as desired.

We note, in particular, that in a basis of orthonormal frames adapted to $T^{*}$,

$$
\Theta_{\alpha}^{\beta} \neq 0 \quad \text { for some } \alpha(\beta) \text { and some } \beta(\alpha) \text {; }
$$

that is, no row or column in the $h \times h$ block is zero.

The local structure of $T^{* \Theta}$ in case the curvature rank is constant is due to Chern and Kuiper [13].

Proposition 6.2. If $h=$ constant on a neighborhood $U$, then $T^{* \Theta}$ is completely integrable on the neighborhood.

PROoF. In orthonormal coframes adapted to $T^{* \Theta}, \Theta_{\lambda}^{j}=0$, and hence the second Bianchi identity yields

$$
0=d \Theta_{\lambda}^{j}+\Sigma \Theta_{\lambda}^{k} \wedge \phi_{k}^{j}-\Sigma \phi_{\lambda}^{i} \wedge \Theta_{i}^{j}=-\Sigma \phi_{\lambda}^{\beta} \wedge \Theta_{\beta}^{j} .
$$

Now by $(6.1) \Theta_{\beta}^{j} \neq 0$ for some $j(\beta)$ and hence $\Theta_{\beta}^{j} \in \Lambda^{2} T^{* \Theta}$ implies $\phi_{\lambda}^{\beta}$ $\in T^{* \Theta}$. As a result we have the Frobenius conditions

$$
d \tau^{\beta}=\Sigma \tau^{\alpha} \wedge \phi_{\alpha}^{\beta}+\Sigma \tau^{\lambda} \wedge \phi_{\lambda}^{\beta} \equiv 0 \bmod T^{* \Theta} .
$$

A similar integer invariant of the curvature matrix at a point $u$ of $M$ is $k(u)$, the smallest dimension of a subspace $W^{*} \subset T^{*}$ such that the linear system $\Theta$ is contained in the exterior ideal generated by the subspace $W^{*}$. The geometric information contained in $k(u)$ most naturally appears in a complementary form and motivates us to introduce the curvature deficiency of $M$ at $u$, which will be denoted by $i(u)$ and is defined by $i(u)=h(u)-k(u)$. These two new integers $k$ and $i$ can also be characterized by a special block structure of the curvature matrix.

Proposition 6.3. The integer $k$ is characterized as the smallest integer, and the integer $i$ is characterized as the largest integer such that there exists a basis of $T^{*}$ 
adapted to $T^{* \Theta}$ with the nontrivial portion of the curvature matrix in the block form

$$
\Theta_{\alpha}^{\beta}=k\{\overbrace{\left(\begin{array}{c|c}
* & * \\
* \underbrace{0}_{i}
\end{array}\right)}\}_{i}, \quad 1 \leqslant \alpha, \beta \leqslant h .
$$

Proof. Let $W^{*} \subset T^{* \Theta}$ have an orthonormal basis $\tau^{1}, \ldots, \tau^{k}$. Then $\Theta_{\alpha}^{\beta}$ $\in$ ideal $W^{*}$ if and only if $R_{\alpha \sigma \tau}^{\beta}=0, k+1 \leqslant \sigma, \tau \leqslant h$, which by the symmetry on pairs of indices holds if and only if $R_{\sigma \alpha \beta}^{\tau}=0$. This last statement is equivalent to the desired conditions $\Theta_{\sigma}^{\tau}=0$.

Let us notice that $\Theta_{\alpha}^{\beta} \in \Lambda^{2} T^{*}$ implies $\Theta_{\alpha}^{\beta} \in \operatorname{ideal}\left(\tau^{1}, \ldots, \tau^{h-1}\right)$ so that $0 \leqslant k \leqslant h-1$. In particular let us note that $h \neq 0$ implies $i \geqslant 1$.

Another obvious integer invariant characterized by a special block structure of the curvature matrix is the integer $q$ equal to the maximal possible number of nonzero blocks in a diagonal block decomposition

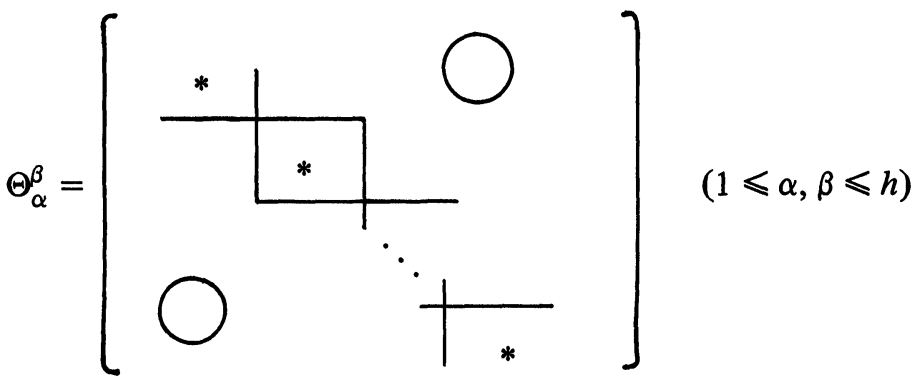

Using the Cartan-Ambrose-Singer holonomy theorem, the integer $q$ is immediately identified with the maximal number of mutually orthogonal subspaces of $T^{* \Theta}$ which are invariant under the restricted holonomy group.

The next result is the local idea underlying the de Rham decomposition.

Proposition 6.4. If the curvature matrix has the form (6.2), then there is an orthogonal decomposition

$$
T^{* \Theta}=T^{* \Theta_{1}} \perp \cdots \perp T^{* \Theta_{q}}
$$

and if the decomposition is constant on a neighborhood, each factor $T^{* \Theta_{1}}$ is completely integrable.

Proof. The first statement follows from the curvature tensor symmetries in a way similar to Proposition 6.1 and the second statement follows from the second Bianchi identities in a way similar to Proposition 6.2.

Finally we note a simple inequality which is of importance in the study of immersions of Riemannian products.

Proposition 6.5. $i \geqslant q$. 
Proof. If we adapt an orthonormal coframe to the orthogonal decomposition $T^{* \Theta}=T^{* \Theta_{1}} \perp \cdots \perp T^{* \Theta_{q}}$ then the curvature matrix has elements in the exterior ideal generated by the elements of the coframe in each factor minus one. Altogether this eliminates $q$ elements from the original $h$ and gives the inequality.

We will now establish a basic dimensional inequality involving the integers $h, s, i$ and $r$. The proof is based on the following unpublished lemma on quadratic exterior equations jointly worked out with M. Kuranishi in 1968.

LEMMA 6.6. Let $V$ be an n-dimensional real vector space and let $y_{\alpha}^{i} \in V$ for $1 \leqslant \alpha \leqslant m \leqslant \operatorname{dim} V$ and $1 \leqslant i \leqslant r$. Then $\sum_{i=1}^{r} y_{\alpha}^{i} \wedge y_{\beta}^{i}=0(1 \leqslant \alpha, \beta$ $\leqslant m$ ) implies $\operatorname{dim}\left\{y_{\alpha}^{i}\right\} \leqslant r$.

Proof. The hypothesis and conclusion are unchanged under $r \times r$ orthogonal transformations $\bar{y}_{\alpha}^{i}=\sum b_{i j} y_{\alpha}^{j}$. As such we may assume $y_{1}^{1}, \ldots, y_{1}^{\rho}$ are linearly independent with $\rho>0$ and $y_{1}^{j}=0(\rho+1 \leqslant j \leqslant r)$, by first reindexing if necessary so that $\operatorname{dim}\left\{y_{1}^{i}\right\}=\rho \neq 0$ and then applying an orthogonal transformation so that

$$
S\left(y_{1}^{1}, \ldots, y_{1}^{r}\right)=\left(\bar{y}_{1}^{1}, \ldots, \bar{y}_{1}^{\rho}, 0, \ldots, 0\right) .
$$

Matters being so, $0=\sum y_{1}^{i} \wedge y_{\beta}^{i}$ implies by Cartan's lemma that

$$
y_{\beta}^{i}=\sum_{j=1}^{\rho} C_{i j}^{\beta} y_{1}^{j} \quad(1 \leqslant i \leqslant \rho) \quad \text { with } C_{i j}^{\beta}=C_{j i}^{\beta} .
$$

Now substitution of these last equations into the hypothesis yields

$$
0=\sum y_{\alpha}^{i} \wedge y_{\beta}^{i}=\sum_{i, j=1}^{\rho} C_{i j}^{\alpha} y_{1}^{i} \wedge y_{\beta}^{j}+\sum_{i=\rho+1}^{r} y_{\alpha}^{i} \wedge y_{\beta}^{i} .
$$

The first of the last two terms, however, vanishes by the symmetry of the $C_{i j}^{\alpha}$ and leaves us with

$$
0=\sum_{i=\rho+1}^{r} y_{\alpha}^{i} \wedge y_{\beta}^{i}, \quad \text { a sum of } r-\rho \text { terms. }
$$

This places us in a position to use induction on $r$. The case $r=1$ being trivial, the general step is given by the inductive hypothesis, "since then

$$
\operatorname{dim}\left\{y_{\alpha}^{i}\right\} \leqslant \operatorname{dim}_{1 \leqslant i \leqslant \rho}\left\{y_{\alpha}^{i}\right\}+\operatorname{dim}_{\rho+1 \leqslant i \leqslant r}\left\{y_{\alpha}^{i}\right\} \leqslant \rho+r-\rho=r .
$$

THeOReM 6.7. Let $X: M_{m} \rightarrow R^{m+p}$ be an isometric immersion. Then at each point $u \in M$, the inequality $0 \leqslant s-h \leqslant r-i$ obtains, where we recall $s=$ rank of the Gauss map, $h=$ rank of the curvature matrix, $r=$ rank of the second fundamental form, and $i=$ the curvature deficiency.

Since these inequalities are of such importance we will make a few historical comments. The inequality with $h=0$ which forces $i=0$ and with $r$ replaced 
by the codimension $p$ is due to E. Cartan [8]. The case with $i=0$ and $r$ replaced by $p$ is due to Chern and Kuiper [13]. The case with $r$ replaced by $p$ and with $i$ replaced by $q$, the number of nontrivial curvature diagonal blocks, is due to Alexander [4]. The case with $r$ replaced by $p$ is equivalent to Alexander and Maltz [5].

Proof. Letting $k=h-i$, we may apply Proposition 6.3 to choose an orthonormal basis $\left\{\tau^{j}\right\}$ for $T^{*}$ and an orthonormal basis $\left\{e_{a}\right\}$ for $N$ such that

$$
\begin{array}{ll}
\Theta_{i}^{j} \in \text { ideal }\left\{\tau^{1}, \ldots, \tau^{k}\right\}, & \\
T^{* \Theta}=\tau^{1}, \ldots, \tau^{h}, & k \leqslant h, \\
T^{* \Phi}=\tau^{1}, \ldots, \tau^{s}, & h \leqslant s, \\
\phi_{i}^{a}=0, & r+1 \leqslant a \leqslant p .
\end{array}
$$

As a result the Gauss equations imply

$$
\sum_{a}\left(\sum h_{i \lambda}^{a} \tau^{\lambda}\right) \wedge\left(\sum h_{j \mu}^{a} \tau^{\mu}\right)=0 \quad(k+1 \leqslant \lambda, \mu \leqslant s) .
$$

If we let $W=\left\{\sum h_{i \lambda}^{a} \tau^{\lambda}\right\}$ and apply the last lemma we find

$$
\operatorname{dim} W \leqslant r .
$$

Now visibly $\left\{\phi_{i}^{a}\right\} \subseteq\left\{\tau^{1}, \ldots, \tau^{k}\right\}+\left\{\sum h_{i \lambda}^{a} \tau^{\lambda}\right\}$; hence $s \leqslant k+\operatorname{dim} W$, but by definition $s-k \geqslant \operatorname{dim} W$, hence

$$
\operatorname{dim} W=s-k=s-h+i .
$$

Finally combining (6.3) and (6.4) gives the final result.

As an immediate example of the impact of this inequality we will prove a result which is important for the analysis of isometric immersions of Riemannian products.

Proposition 6.8. Let $X: M_{m} \rightarrow R^{m+p}$ be an isometric immersion of a Riemannian metric with curvature deficiency everywhere equal to $p$. Then for all $u \in M$

(1) $\operatorname{dim} O_{u}^{1}=m+p$, hence is constant, and

(2) $s=h$, hence the rank of the Gauss map is intrinsic.

PRoof. $0 \leqslant s-h \leqslant r-i \leqslant p-i=p-p=0$; hence $r=i=p$ and $s=h$.

In the case that $h<s$, there is additional information on the immersion at a point based on the fact that $\Theta_{i}^{j} \in \Lambda^{2} T^{* \Theta}$. In the notation of the last theorem this implies

$$
\sum\left(h_{i \lambda}^{a} h_{j \beta}^{a}-h_{i \beta}^{a} h_{j \lambda}^{a}\right) \tau^{\lambda} \wedge \tau^{\beta}=0, \quad 1 \leqslant \beta \leqslant h, h+1 \leqslant \lambda \leqslant s .
$$

We have not been able to extract the geometric information in this last set of equations in terms of an inequality on the basic integers of the immersion.

We may get some global results from these pointwise estimates, by using the 
compactness to guarantee the existence of at least one point where $s=m$. The first theorem sharpens a result in [13].

THEOREM 6.9. Let $M_{m}$ be a compact Riemannian manifold with a nonzero sectional curvature at each point. Then there exists no isometric immersion with $p<m+1-\max _{u} h(u)$.

Proof. By Proposition 4.4 there is a point at which $s=m$. Hence at this point $0 \leqslant m-h \leqslant p-i$. Now the curvature hypothesis implies that $h \geqslant 2$ everywhere and hence that $i \geqslant 1$. Thus $p \geqslant m-h+1 \geqslant m+1-\max _{u} h$.

A much more dramatic result is obtained in the case of curvature identically zero. This result is due to Tompkins [28].

THEOREM 6.10. Let $M_{m}$ be a compact Riemannian manifold having zero curvature matrix. Then there is no isometric immersion in $R^{2 m-1}$.

Proof. Since $h=0, i=0$ and using the point at which $s=m$ which is guaranteed by Proposition 4.4 we see that $0 \leqslant m \leqslant r \leqslant p$ as desired.

Finally we introduce an increasing sequence of integer invariants of the curvature matrix related to a canonical filtration of the linear system $\Theta$.

The $t^{\text {th }}$ trace rank of $M$ at a point $u$ is defined to be the smallest integer $\rho_{t}$ such that the metric trace

$$
\left(\operatorname{tr}_{\mathrm{I}} A \Theta\right)^{\rho_{t}+1}=\left(\sum a_{i j} \Theta_{i}^{j}\right)^{\rho_{t}+1}=0
$$

for all real $m \times m$ matrices $A$ of rank $\leqslant t$.

Since the conditions become increasingly less restrictive,

$$
0 \leqslant \rho_{1} \leqslant \cdots \leqslant \rho_{m} \leqslant[m / 2]+1 .
$$

As usual in such constructions, the first and last trace rank are the easiest to analyze.

The top trace rank $\rho_{m}$ has a simple alternate description which leads us to give this trace rank the special name of curvature wedge length. In order to see the reason for this nomenclature we note that the smallest integer such that $\left(\sum a_{i j} \Theta_{i}^{j}\right)^{\rho_{m}+1}=0$ is the same as the smallest integer such that $\pi^{\rho_{m}+1}=0$ for all $\pi \in \Theta$. Now let us define a linear mapping

$$
\downarrow_{q}: \bigcirc{ }^{q} \Theta \rightarrow \Lambda^{2 q} T^{* \Theta}
$$

by covering the symmetric multilinear mapping

$$
\eta_{q}: \Theta \times \therefore \times \Theta \rightarrow \Lambda^{2 q} T^{* \Theta}
$$

defined by

$$
\eta_{q}\left(\pi^{1}, \ldots, \pi^{q}\right)=\pi^{1} \wedge \cdots \wedge \pi^{q} .
$$

Now we see that the condition $\pi^{\rho_{m}+1}=0$ for all $\pi \in \Theta$ is equivalent to 
$l_{\rho_{m}+1}=0$ and this holds if and only if $\eta_{\rho_{m}+1}=0$ since a symmetric multilinear map is determined by its associated polynomial. As a result the condition that the curvature wedge length be $\rho_{m}$ is precisely the condition that the exterior product of any $\left(\rho_{m}+1\right)$ elements of $\Theta$ be zero.

An easy lower bound for $\rho_{m}$ can be obtained by considering the traces of the matrix product of the curvature matrix with itself $q$-times. If $q$ is the largest integer such that $\operatorname{Tr}_{\mathrm{I}} \Theta^{q} \neq 0$, then this is a nonzero sum of $q$-fold products of elements from $\Theta$ and, hence, $\rho_{m} \geqslant q$. This particular computation was chosen since if $q=2 k$, then

$$
\operatorname{tr}_{\mathrm{I}} \Theta^{q}=P_{k}(\Theta),
$$

the Chern-Weil representative $4 k$-form for the $k$ th Pontryagin class [11].

A particularly fortuitous situation occurs if $h=2 \rho_{m}$. Then

$$
l_{h}: \rho^{\rho_{m}} \theta \rightarrow \Lambda^{h} T^{* \Theta} \simeq R
$$

becomes a nonzero symmetric $p$-linear form, that is a polynomial. The invariants of this polynomial then lead to special classifications of curvature tensors. As a particular example of this we note that if $M$ is a four manifold then $l_{2}: \bigcirc^{2} \Theta \rightarrow \Lambda^{4} T^{*} \simeq R$ is a symmetric bilinear form which is the restriction of the symmetric bilinear form $\left(\Lambda^{2} T^{*}\right) \times\left(\Lambda^{2} T^{*}\right) \rightarrow \Lambda^{4} T^{*} \simeq R$ defined by exterior multiplication. This is easily seen to have rank 6 and index 3 . The resulting classification of the curvature tensor has been used by physicists for years and is known as the Petrov classification [24].

The bottom trace rank $\rho_{1}$ is easiest to analyze when we note that a matrix $a_{i j}$ has rank one if and only if it has the form $a_{i j}=x_{i} y_{j}$. This may be seen via the isomorphism Hom $(V, V) \simeq V \otimes V^{*}$ under which the rank one matrices go into monomials $x \otimes y^{*}$. As a result $\rho_{1}$ is characterized as the smallest integer such that

$$
\left(\sum x_{i} y_{j} \Theta_{i}^{j}\right)^{\rho_{1}+1}=0 \text { for all } x_{i}, y_{j} \in R .
$$

We remark that the statement $\rho_{1} \geqslant q$ is the same as J. D. Moore's condition $A(q)$ [22].

Our next result which shows that $m+\rho_{1}$ is an intrinsic lower bound for the dimension of an osculating space at $u$ leads us to give the trace rank $\rho_{1}$ the special name of osculation degree.

Proposition 6.11. Let $X: M_{m} \rightarrow R^{m+p}$ be an isometric immersion of a Riemannian manifold. Then for every point $u \in M_{m}, r(u) \geqslant \rho_{1}(u)$.

Proof. Given $x_{i}, y_{j} \in R$, then by the Gauss equations

$$
-\sum x_{i} y_{j} \Theta_{i}^{j}=\sum x_{i} y_{j} \phi_{1}^{a} \wedge \phi_{j}=\sum\left(\sum x_{i} \phi_{i}^{a}\right) \wedge\left(\sum y_{j} \phi_{j}^{a}\right),
$$

which with the usual choice of normal frames, has the form 


$$
\sum_{a=m+1}^{m+r} \omega^{a} \wedge \eta^{a}
$$

As such

$$
\left(\sum x_{i} y_{j} \Theta_{i}^{j}\right)^{r+1}=\left(\sum_{a=m+1}^{m+r} \omega^{a} \wedge \eta^{a}\right)^{r+1}=0 .
$$

This implies $\rho_{1}(u) \leqslant r(u)$ as required.

Necessary and sufficient conditions that the equality $r=\rho_{1}$ occurs will be given in $\S 8$.

COROllaRY 6.12. If $X: M_{m} \rightarrow R^{m+p}$ is an isometric immersion of a Riemannian manifold with osculation degree $\rho_{1}=p$ everywhere, then the dimension of all osculating spaces is constantly equal to $m+p$.

Proposition 6.13. Let $M \times N$ be a Riemannian product. Then

$$
\rho_{1}(M \times N)=\rho_{1}(M)+\rho_{1}(N) .
$$

Proof. The identity follows from the isomorphism

$$
\Lambda^{p} T^{*}(M) \oplus T^{*}(N) \simeq \underset{i+j=p}{\oplus} \Lambda^{i} T^{*}(M) \otimes \Lambda^{j} T^{*}(N)
$$

and the fact that an orthonormal basis may be chosen so that the curvature matrix of a product metric satisfies

$$
\begin{aligned}
& \sum x_{i} y_{j} \Theta_{i}^{j}=\sum x_{\alpha} y_{\beta} \Theta_{\alpha}^{\beta}+\sum x_{\lambda} y_{\mu} \Theta_{\lambda}^{\mu} \\
&(1 \leqslant \alpha, \beta \leqslant m, m+1 \leqslant \lambda, \mu \leqslant m+n) \\
& \subseteq \Lambda^{2} T^{*}(M) \oplus \Lambda^{2} T^{*}(N) .
\end{aligned}
$$

7. The Gauss sequence. We define a linear mapping

$$
\beta:\left(T^{*} \otimes N\right) \otimes\left(T^{*} \otimes N\right) \rightarrow \Lambda^{2} T^{*}
$$

to be the tensor product of exterior multiplication $T^{*} \otimes T^{*} \rightarrow T^{*}$ and the scalar product $N \otimes N \rightarrow R$. Thus if

$$
\phi_{i}=\sum m_{i j}^{a} \tau^{j} \otimes e_{a} \text { and } \psi_{i}=\sum n_{i k}^{b} \tau^{k} \otimes e_{b}
$$

then

$$
\beta\left(\Sigma \phi_{i} \otimes \psi_{i}\right)=\frac{1}{2} \sum\left(m_{i j}^{a} n_{i k}^{a}-m_{i k}^{a} n_{i j}^{a}\right) \tau^{j} \wedge \tau^{k} .
$$

If we restrict to $\Phi \otimes \Phi$, then a typical element has the form

$$
\sum \phi_{i} \otimes \psi_{i}=\sum m_{i j} \phi_{j}^{a} \otimes e_{a} \otimes \sum n_{i k} \phi_{k}^{b} \otimes e_{b},
$$


and hence by the Gauss equations

$$
\beta\left(\sum \phi_{i} \otimes \psi_{i}\right)=-\sum m_{i j} n_{i k} \Theta_{j}^{k} .
$$

This shows there is a short exact sequence $0 \rightarrow K \rightarrow \Phi \otimes \Phi \rightarrow \Theta \rightarrow 0$ which we call the Gauss sequence. The basic relations between the extrinsic geometry reflected in the linear system $\Phi$ and the intrinsic geometry reflected in the linear system $\Theta$ come from the careful study of this sequence.

In general, we will analyze the sequence through the study of the associated alternating bilinear map $\beta:\left(T^{*} \otimes N\right) \times\left(T^{*} \otimes N\right) \rightarrow \Lambda^{2} T^{*}$.

A subspace $\Psi \subset T^{*} \otimes N$ is called orthogonally reducible with respect to $\beta$ if there is a nontrivial decomposition $\Psi=\Psi_{1} \oplus \Psi_{2}$ satisfying $\beta(\phi, \psi)=0$ for all $\phi \in \Psi_{1}$ and $\psi \in \Psi_{2}$. We will denote such a reduction by $\Psi=\Psi_{1} \perp \Psi_{2}$.

If there is no such decomposition, then $\Psi$ is said to be orthogonally irreducible with respect to $\beta$.

We define $\Phi^{0}$ to be the conjugate subsystem of $\Phi$ under $\beta$. That is,

$$
\Phi^{0}=\{\phi \in \Phi \mid \beta(\phi, \psi)=0 \text { for all } \psi \in \Phi\} .
$$

Since any complement $\Phi^{\prime}$ of $\Phi^{0}$ has the property that $\Phi=\Phi^{0} \perp \Phi^{\prime}$ we may choose a complement $\Phi^{\prime}$ and an orthonormal basis such that

$$
\Phi^{\prime}=\left\{\Sigma \phi_{1}^{a} \otimes e_{a}, \ldots, \Sigma \phi_{h}^{a} \otimes e_{a}\right\}
$$

where $h$ is the curvature rank, and

$$
\Phi^{0}=\left\{\Sigma \phi_{h+1}^{a} \otimes e_{a}, \ldots, \Sigma \phi_{s}^{a} \otimes e_{a}\right\} .
$$

If $\Phi=\Phi^{0}$ then II is said to be an exterior orthogonal system of quadratic forms. This case was studied in detail by E. Cartan [8].

Proposition 7.1. The bilinear mapping $\beta: \Phi \otimes \Phi \rightarrow \Theta$ is essential if and only if $h=s$.

Proof. $\beta$ is essential if and only if

$$
\Phi^{0}=\left\{\Sigma \phi_{h+1}^{a} \otimes e_{a}, \ldots, \Sigma \phi_{s}^{a} \otimes e_{a}\right\}=0 ;
$$

hence if and only if $s=h$.

If $s=h=$ constant so that $\beta$ is essential, then necessarily $\Phi^{0}=0$. This is the critical calculation for what are called cylindricity theorems. An example will be discussed in $\$ 9$.

We say that a subspace $\psi \subset \Phi^{\prime}$ is maximal totally isotropic if it is a subspace of $\Phi^{\prime}$ of maximal dimension on which the restriction of $\beta$ is identically zero. The problem of studying the curvature deficiency of an induced metric is exactly equivalent to studying the dimension of a maximal totally isotropic subspace of $\Phi^{\prime}$. In order to see this let $\Phi_{M^{\prime}}$ be a maximal totally isotropic subspace of $\Phi^{\prime}$, and let $\Phi_{M^{\prime}}$ have basis $\left\{\phi^{k+1}, \ldots, \phi^{h}\right\}$. By Proposition 4.2 we may choose an orthonormal base so that 


$$
\Phi_{M^{\prime}}=\left\{\sum \phi_{k+1}^{a} \otimes e_{a}, \ldots, \Sigma \phi_{h}^{a} \otimes e_{a}\right\},
$$

and then for $k+1 \leqslant \sigma, \tau \leqslant h$,

$$
-\Theta_{\sigma}^{\tau}=\Sigma \phi_{\sigma}^{a} \wedge \phi_{\tau}^{a}=\beta\left(\Sigma \phi_{\sigma}^{a} \otimes e_{a}, \Sigma \phi_{\tau}^{b} \otimes e_{b}\right)=0 .
$$

Conversely, if the curvature deficiency is $i$, then using the orthonormal frames guaranteed by Proposition 6.3 we see that

$$
\Phi_{M^{\prime}}=\left\{\sum \phi_{k+1}^{a} \otimes e_{a}, \ldots, \Sigma \phi_{h}^{a} \otimes e_{a}\right\}
$$

is a maximal totally isotropic subspace of $\Phi^{\prime}$.

Proposition 7.2. $\Phi^{\prime}=\psi^{1} \perp \cdots \perp \psi^{q}$ with $\psi^{k} \neq 0(1 \leqslant k \leqslant q)$ implies that the curvature deficiency $i \geqslant q$.

Proof. Let $\phi^{k}$ be nonzero elements in $\psi^{k}(1 \leqslant k \leqslant q)$. Then the subspace $\left\{\phi^{1}, \ldots \phi^{q}\right\}$ of $\Phi^{\prime}$ is totally isotropic. Hence $i \geqslant q$.

COROLlaRY 7.3. If the curvature deficiency is one, then $\Phi^{\prime}$ is orthogonally irreducible with respect to $\beta$.

8. Allendoerfer type number. Let $X: M_{m} \rightarrow R^{m+p}$ and let $E_{q}$ be a $q$-plane field defined in a neighborhood of a point $u \in M_{m}$. If $x_{1}, \ldots, x_{q}$ is a basis for $E_{q}$ so that $E_{q}=x_{1} \wedge \cdots \wedge x_{q}$ in the sense of Plücker geometry [25, p. 16], then we define the normal twist of $E_{q}$ at $u$ to be $\left.\operatorname{dim}\left\{x_{\alpha}\right\lrcorner \mathrm{II}^{a}\right\}(1 \leqslant \alpha \leqslant q$, $m+1 \leqslant a \leqslant m+p)$.

Since the $\left.\operatorname{span}\left\{x_{\alpha}\right\lrcorner \mathrm{II}^{a}\right\}$ is unchanged under nonsingular transformations of $x_{1}, \ldots, x_{q}$ we may choose local frames so that $E_{q}=e_{1} \wedge \cdots \wedge e_{q}$. Since

$$
\begin{aligned}
& d\left(e_{1} \wedge \cdots \wedge e_{q}\right) \\
& \quad=\Sigma e_{1} \wedge \cdots \wedge e_{\alpha-1} \wedge\left(\Sigma \phi_{\alpha}^{\beta} e_{\beta}+\sum \phi_{\alpha}^{a} e_{a}\right) \wedge e_{\alpha+1} \wedge \cdots \wedge e_{q}
\end{aligned}
$$

and the normal twist at $u$ equals $\operatorname{dim}\left\{\phi_{\alpha}^{a}\right\}(1 \leqslant \alpha \leqslant q, m+1 \leqslant a \leqslant m+p)$, we see that the normal twist may be geometrically interpreted as the number of independent parameters needed to locally describe the nontangential motion of the $q$-plane field.

The Allendoerfer type number of an immersion of $M$ at $u$ is the largest integer $\tau$ such that there is a $\tau$-plane field defined in a neighborhood of $u$ with normal twist equal to $r \tau$, where $r$ is the rank of II.

We note that the value $r \tau$ is the maximal possible value for the normal twist of a $\tau$-plane field since $\operatorname{dim}\left\{\phi_{\alpha}^{a}\right\} \leqslant r \tau$.

A consequence of our calculation with local frames adapted to the plane field is that the type number is $\tau$ if and only if there is a choice of local frames so that the $r \tau$ 1-forms $\phi_{1}^{a}, \ldots, \phi_{\tau}^{a}$ are all linearly independent and $\tau$ is maximal with respect to this last property. The existence of an immersion with type number $\tau$ implies that the osculating spaces are not very large. In fact since $r \tau \leqslant s \leqslant m$ we see that $\max _{u} \operatorname{dim} O_{u}^{1} \leqslant m+[m / \tau]$. 
In the simplest case of hypersurfaces $X: M_{m} \rightarrow R^{m+1}$ the type number $\tau=\operatorname{dim}\left\{\phi_{\alpha}^{m+1}\right\}$, which is just the matrix rank of the second fundamental form II.

The immersions with type number everywhere $\leqslant 2$ are of particular interest since the geometry is quite rigid. We begin by showing that this class of immersions has a simple characterization.

Proposition 8.1. Let $X: M_{m} \rightarrow R^{m+p}$. Then $\tau(u) \geqslant 2$ if and only if the osculation degree $\rho_{1}(u)=r(u)$.

Proof. If $\rho_{1}=r$ then there exist real numbers $x_{i}, y_{j}$ such that $\left(\sum x_{i} y_{j} \Theta_{i}^{j}\right)^{r}$ $\neq 0$. The Gauss equations then imply

$$
0 \neq\left(\sum\left(\sum x_{i} \phi_{i}^{a}\right) \wedge\left(\sum y_{j} \phi_{j}^{a}\right)\right)^{r},
$$

and hence that $\sum x_{i} \phi_{i}^{a}$ and $\sum y_{j} \phi_{j}^{a}$ define $2 r$ linearly independent 1-forms. If we introduce $X=\sum x_{i} e_{i}$ and $Y=\sum y_{j} e_{j}$ then the 2-plane field $X \wedge Y$ has normal twist $2 r$.

Conversely if $\tau \geqslant 2$, we may choose orthonormal frames so that $\phi_{1}^{a}$ and $\phi_{2}^{a}$ are $2 r$ linearly independent 1 -forms, and, hence, so that

$$
\left(-\Theta_{1}^{2}\right)^{r}=\left(\Sigma \phi_{1}^{a} \wedge \phi_{2}^{a}\right)^{r} \neq 0 .
$$

This implies that $\rho_{1} \geqslant r$, but Proposition 6.11 implies that $r \geqslant \rho_{1}$ which establishes the equality.

Recalling Proposition 6.13 that the osculation degree of a Riemannian product is the sum of the osculation degrees of the factor; we note that one can construct high codimension immersions of type number greater than or equal to two by forming cartesian products of hypersurface immersions with second fundamental forms of matrix rank greater than or equal to two.

COROLlaRY 8.2. Let

$$
M_{m} \stackrel{X}{\underset{X^{\#}}{\longrightarrow}} R^{m+p}
$$

be isometric immersions of a Riemannian manifold $M_{m}$. If $\tau(X) \geqslant 2$ at a point $u$, then the rank of $X^{\#}=r^{\#}(u) \geqslant r(u)=$ the rank of $X$.

Proof. Since $\tau \geqslant 2, r=\rho_{1}$. Hence by Proposition 6.11 applied to the $X^{\#}$ immersion we have $r^{\#} \geqslant \rho_{1}=r$.

CoROllary 8.3. Let $X: M_{m} \rightarrow R^{m+p}$ be an immersion with $\tau(X) \geqslant 2$. Then $\max _{u} \operatorname{dim} O_{u}^{1}=p$ implies there is no isometric immersion in $R^{m+p-1}$.

If the type number is $\geqslant 2$ we have seen that we may choose frames so that $\phi_{1}^{a}, \phi_{2}^{a}$ are linearly independent for $m+1 \leqslant a \leqslant m+r$. These forms give rise to two elements, 


$$
\phi_{1}=\Sigma \phi_{1}^{a} \otimes e_{a} \text { and } \phi_{2}=\Sigma \phi_{2}^{a} \otimes e_{a},
$$

in the linear system $\Phi$. The existence of these elements is one of the principal tools in the analysis of these immersions.

LeMma 8.4. Let $X: M_{m} \rightarrow R^{m+p}$ be an immersion with $\tau \geqslant 2$. Then the equations

(1) $\beta\left(\phi_{1}, \psi\right)=0$ and

(2) $\beta\left(\phi_{2}, \psi\right)=0$ with $\psi \in T^{*} \oplus$ imply $\psi=0$.

Proof. Equation (1) and Cartan's lemma imply $T^{* \psi} \subset\left\{\phi_{1}^{a}\right\}$ and equation (2) implies $T^{* \psi} \subset\left\{\phi_{2}^{a}\right\}$. Hence by linear independence, $T^{* \psi}=0$.

Proposition 8.5. Let $X: M_{m} \rightarrow R^{m+p}$ be an immersion with $\tau \geqslant 2$. Then the bilinear mapping $\beta: \Phi \times \Phi \rightarrow \Theta$ is essential, and as a result the rank of the Gauss map is intrinsic.

Proof. If $\psi \in \Phi$ with $\beta(\phi, \psi)=0$ for all $\phi \in \Phi$ then we may take $\phi=\phi_{1}$ and $\phi=\phi_{2}$ and apply the last lemma to deduce $\psi=0$. This implies that $\beta$ is essential and, hence, Proposition 7.1 implies that $s=h$.

Now we consider local results that depend on $r$, and hence the dimension of the osculating spaces, being constant. The first result was found by Allendoerfer [2] when $\tau \geqslant 3$ and independently by Spivak [25, Vol. 4] and Erbacher [14] when $\tau=2$.

Proposition 8.6. Let $X: M_{m} \rightarrow R^{m+p}$ have type number $\tau \geqslant 2$ and constant dimensional osculating spaces. Then all osculating spaces are parallel and as a result $X: M_{m} \rightarrow R^{m+r}$.

Proof. Since $r$ is constant we may choose frames so that

$$
\phi_{i}^{c}=0 \quad(m+r+1 \leqslant c \leqslant m+p, 1 \leqslant i \leqslant m)
$$

and $\phi_{1}^{a}, \phi_{2}^{a}$ are linearly independent for $m+1 \leqslant a \leqslant m+r$.

The structure equations imply

$$
\begin{aligned}
& 0=d \phi_{\alpha}^{c}=\sum \phi_{\alpha}^{\beta} \wedge \phi_{\beta}^{c}+\sum \phi_{\alpha}^{a} \wedge \phi_{a}^{c}+\sum \phi_{\alpha}^{e} \wedge \phi_{e}^{c}=\sum \phi_{\alpha}^{a} \wedge \phi_{a}^{c}, \\
& 1 \leqslant \alpha, \beta \leqslant m, \quad m+1 \leqslant a, b \leqslant m+r, \quad m+r+1 \leqslant c, e \leqslant m+p .
\end{aligned}
$$

As a result $\beta\left(\phi, \phi_{a}^{c}\right)=0$ for all $\phi \in \Phi$, and Proposition 8.5 implies $\phi_{a}^{c}=0$. Now these equations force $d\left(e_{1} \wedge \cdots \wedge e_{m+r}\right)=0$, and hence imply that all the osculating spaces are parallel. This means that the minimal enveloping spaces $\mathscr{E}\left(M_{m}\right)$ of $\S 3$ has dimension $m+r$ and hence that $X: M_{m} \rightarrow R^{m+r}$.

Our next results require an algebraic lemma due to Chern [12].

LemMA 8.7. Let $y_{\alpha}^{a}$ and $\bar{y}_{\alpha}^{a} \in V(1 \leqslant a \leqslant r, 1 \leqslant \alpha \leqslant n)$ an m-dimensional real vector space. Then if 


$$
\sum_{a=1}^{r} y_{\alpha}^{a} \wedge y_{\beta}^{a}=\sum_{a=1}^{r} \bar{y}_{\alpha}^{a} \wedge \bar{y}_{\beta}^{a}
$$

where $y_{1}^{a}, y_{2}^{a}, y_{3}^{a}$ are a set of $3 r$ linearly independent vectors, then there exists an $r \times r$ orthogonal matrix $b_{a c}$ such that

$$
y_{\alpha}^{a}=\sum b_{a c} \bar{y}_{\alpha}^{c} \quad(1 \leqslant \alpha \leqslant n) .
$$

Proof. $0 \neq\left(\sum_{a=1}^{r} y_{\alpha}^{a} \wedge y_{\beta}^{a}\right)^{r}=\left(\sum_{a=1}^{r} \bar{y}_{\alpha}^{a} \wedge \bar{y}_{\beta}^{a}\right)^{r}$ for the distinct pairs $1 \leqslant \alpha, \beta \leqslant 3$. This implies that $\left\{\bar{y}_{\alpha}^{a}\right\}$ are linearly independent sets and that the subspaces $\left\{y_{\alpha}^{a}\right\}=\left\{\bar{y}_{\alpha}^{a}\right\}$ for $1 \leqslant \alpha \leqslant 3$.

Now let $U=\left\{\bar{y}_{1}^{a}\right\}, V=\left\{\bar{y}_{2}^{a}\right\}$ and $W=\left\{\bar{y}_{3}^{a}\right\}$ and let

$$
\bar{y}_{1}^{a}=\sum B_{a c} y_{1}^{c}, \quad \bar{y}_{2}^{a}=\sum C_{a b} y_{2}^{b}, \quad \bar{y}_{3}^{a}=\sum D_{a b} y_{3}^{b} .
$$

Then relative to the above basis of $U \oplus V$,

$$
\left(\begin{array}{cc}
B_{a c} & 0 \\
0 & C_{a c}
\end{array}\right)
$$

is a symplectic matrix and as a result $\mathrm{I}={ }^{t} B C$. Similarly $\mathrm{I}={ }^{t} C D$ and $\mathrm{I}={ }^{t} B D$ which implies $B=C=D$ and $B$ is orthogonal.

Now the identity

$$
\sum \bar{y}_{\alpha}^{a} \wedge \bar{y}_{\beta}^{a}=\sum B_{a c} y_{\alpha}^{c} \wedge \bar{y}_{\beta}^{a}=\sum y_{\alpha}^{c} \wedge B_{a c} \bar{y}_{\beta}^{a}
$$

subtracted from (8.1) for $\alpha=1,2,3$ gives

$$
0=\sum y_{\alpha}^{a} \wedge\left(y_{\beta}^{a}-\sum B_{c a} \bar{y}_{\beta}^{c}\right)
$$

and, hence, by the linear independence of the sets $\left\{y_{\alpha}^{a}\right\}(1 \leqslant \alpha \leqslant 3)$ we have

$$
y_{\beta}^{a}=\sum B_{c a} \bar{y}_{\beta}^{c}
$$

and we may take $b_{a c}=B_{c a}$ as claimed.

Proposition 8.8. Let

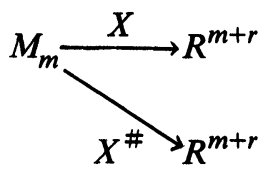

be isometric immersions of a Riemannian manifold $M_{m}$ where $\tau(X) \geqslant 2$ and $r(X)=r=$ constant. Then

$$
\tau(X)=\tau\left(X^{\#}\right) \text { and } r^{\#}=r .
$$

Proof. Since $\tau(X) \geqslant 2$ we know by Proposition 8.1 that $\rho_{1}=r$. Then by Proposition $6.11 r \geqslant r^{\#} \geqslant r$. Hence $r^{\#}=r=\rho_{1}$ and again by Proposition 8.1 we deduce $\tau\left(X^{\#}\right) \geqslant 2$. 
Now if $\tau(X)$ and $\tau\left(X^{\#}\right)$ are both equal to two, we are done. If either $\tau(X)$ or $\tau\left(X^{\#}\right)$ is greater than two we may apply the last lemma to find an orthonormal basis with $\phi_{i}^{a}=\phi_{i}^{a \#}$ and, hence, necessarily $\tau(X)=\tau\left(X^{\#}\right)$.

Our final results are slight refinements of theorems due to Allendoerfer [2].

THEOREM 8.9. Let

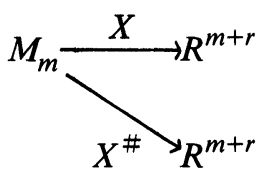

be isometric immersions of a Riemannian manifold $M_{m}$ where $\tau(X) \geqslant 2, r(X)$ $=r=$ constant and $\mathrm{II}=\mathrm{II}^{\#}$. Then $X$ and $X^{\#}$ differ by at most a reflection and a rigid motion.

Proof. By hypothesis we may apply a reflection if necessary to find orthonormal frames for which

$$
\tau^{i}=\tau^{i \#}, \quad \phi_{i}^{j}=\phi_{i}^{j \#}, \quad \phi_{i}^{a}=\phi_{i}^{a \#} .
$$

Hence the Codazzi-Mainardi equations

$$
\begin{aligned}
d \phi_{i}^{a} & =\sum \phi_{i}^{j} \wedge \phi_{j}^{a}+\sum \phi_{i}^{b} \wedge \phi_{b}^{a}, \\
d \phi_{i}^{a \#} & =\sum \phi_{i}^{j \#} \wedge \phi_{j}^{a \#}+\sum \phi_{i}^{b \#} \wedge \phi_{b}^{a \#}
\end{aligned}
$$

imply $\sum \phi_{i}^{b} \wedge\left(\phi_{b}^{a}-\phi_{b}^{a \#}\right)=0$ which, since $\tau(X) \geqslant 2$, implies

$$
\phi_{b}^{a}=\phi_{b}^{a \#} .
$$

Equations (8.2) and (8.3) then imply uniqueness up to euclidean motions [25].

Corollary 8.10. Let

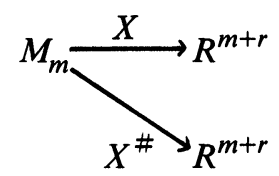

be isometric immersions of a Riemannian manifold where $\tau(X) \geqslant 3$ and $r(X)$ $=r=$ constant. Then $X$ and $X^{\#}$ are unique up to reflection and rigid motions.

Proof. Since $\tau(X) \geqslant 3$ we may apply Lemma 8.7 to deduce that after a possible reflection and rigid motion II $=\mathrm{II}^{\#}$, and hence the result follows by the last theorem.

The result is false for $\tau=2$ with the classical bending of a piece of the sphere [25, Vol. 5] serving as a counterexample.

Unfortunately, I know of no global theorems involving the type number. Since the class of immersions with $\tau \geqslant 2$ has a simple characterization and 
has a quite rigid geometry, an interesting problem would be to classify those Riemannian manifolds which admit an immersion with $\tau(X) \geqslant 2$.

9. Isometric immersions of Riemannian products. If $X_{M}: M_{m} \rightarrow R^{m+p}$ and $X_{V}: V_{n} \rightarrow R^{n+q}$ are isometric immersions of Riemannian manifolds, then the product immersion $X_{M} \times X_{V}: M_{m} \times V_{n} \rightarrow R^{m+n+p+q}$ is an isometric immersion of the Riemannian product.

A natural question and a topic of recent interest is the study of when an isometric immersion of a Riemannian product $X: M_{m} \times V_{n} \rightarrow R^{m+n+p}$ is a product of isometric immersions. If this occurs, the immersion is called reducible.

We make the index conventions for this section that

$$
\begin{array}{lrl}
1 \leqslant \alpha, \beta, \gamma \leqslant m, & m+1 \leqslant \lambda, \mu, \eta \leqslant m+n, \\
1 \leqslant i, j, k \leqslant m+n, & m+n+1 \leqslant a, b, c \leqslant m+n+p,
\end{array}
$$

and the convention on local frame fields that $\left\{e_{\alpha}\right\}$ are tangent to $M_{m} \times q$ for all $q \in V_{n}$ and $\left\{e_{\lambda}\right\}$ are tangent to $p \times V_{n}$ for all $p \in M_{m}$. With this choice the product metric satisfies $\phi_{\alpha}^{\lambda}=0$ and $\Theta_{\alpha}^{\lambda}=0$. The Gauss equations then induce an orthogonal decomposition with respect to $\beta, \Phi=\Psi_{M} \perp \Psi_{V}$, where

$$
\Psi_{M}=\left\{\phi_{\alpha}^{a} \otimes e_{a}\right\} \quad \text { and } \quad \Psi_{V}=\left\{\phi_{\lambda}^{a} \otimes e_{a}\right\} .
$$

The minimal enveloping subspaces

$$
T^{* \Psi_{M}}=\left\{\sum h_{\alpha i}^{a} \tau^{i}\right\} \quad \text { and } \quad T^{* \Psi_{\nu}}=\left\{\sum h_{\lambda j}^{a} \tau^{j}\right\}
$$

can then be used to give a simple local criterion for reducibility.

Proposition 9.1. Let $X: M_{m} \times V_{n} \rightarrow R^{m+n+p}$ be an isometric immersion of a connected Riemannian product. Then $X$ is reducible if and only if $T^{* \Psi_{M}} \subset T^{*}(M)$ and $T^{* \Psi_{V}} \subset T^{*}(V)$.

In terms of components these last conditions are equivalent to $h_{\alpha \lambda}^{a}=0$ which implies that the second fundamental form is simultaneously diagonalized in block diagonal form

$$
\mathrm{II}=\Sigma\left(\frac{*}{0}|0|\right)^{a} e_{a}
$$

In Moore [21] it is shown that having the second fundamental form simultaneously diagonalized in this fashion implies that an immersion is reducible. We now give a more geometric refinement of this result and a new proof using a minimal enveloping subspace idea.

Proposition 9.2. Let $X: M_{m} \times V_{n} \rightarrow R^{m+n+p}$ be an isometric immersion of a connected Riemannian product. Then $X$ is reducible if and only if the instantaneous mean curvature vectors 


$$
H_{M}=\sum h_{\alpha \alpha}^{a} e_{a} \text { and } H_{V}=\sum h_{\lambda \lambda}^{a} e_{a}
$$

are perpendicular.

Proof. The condition is clearly necessary. In order to see that it is sufficient we first note that $H_{M} \cdot H_{V}=0$ if and only if

$$
\sum h_{\alpha \alpha}^{a} h_{\lambda \lambda}^{a}=0 .
$$

Now by the Gauss equations, $\Theta_{\alpha}^{\lambda}=0$ implies

$$
0=\sum h_{\alpha i}^{a} h_{\lambda j}^{a}-h_{\alpha j}^{a} h_{\lambda i}^{a}
$$

and, hence, taking $i=\lambda$ and $j=\alpha$ and applying (8.1) we have

$$
h_{\alpha \lambda}^{a}=0 \text {. }
$$

This in turn implies that the normal component of the instantaneous osculating spaces

$$
N\left(O_{u}^{1}(M)\right)=\left\{\sum h_{\alpha \beta}^{a} e_{a}\right\} \quad \text { and } \quad N\left(O_{u}^{1}(V)\right)=\left\{\sum h_{\lambda \mu}^{a} e_{a}\right\}
$$

are perpendicular for each $u \in M \times V$.

In addition, (8.2) implies

$$
\begin{aligned}
d\left(e_{1} \wedge \cdots \wedge e_{m}\right) & =\sum \phi_{\alpha}^{a} e_{1} \wedge \cdots \wedge e_{\alpha-1} \wedge e_{a} \wedge e_{\alpha+1} \wedge \cdots \wedge e_{m} \\
& \equiv 0 \bmod \left(\tau^{1}, \ldots, \tau^{m}\right)
\end{aligned}
$$

which implies that the restriction maps $X_{M_{m}} \times q: M_{m} \rightarrow R^{m+n+p}$ for $q \in N$ are independent of the differentials of the coordinates on $N$ and hence are independent of $q$. This now gives us a unique restriction map $\left.X\right|_{M}$. In addition, the last computation shows that the minimal enveloping subspace of the linear system $\left\{\sum \phi_{\alpha}^{a} \otimes e_{a}\right\}$ given by $\left\{\sum h_{\alpha \beta}^{a} e_{a}\right\}$ is independent of $q$ and this is the normal component of the first osculating space of the immersion $\left.X\right|_{M}$.

Mutatis mutandis the same results hold for the second factor. That is, there is a unique restriction map $\left.X\right|_{V}$ with normal component of the first osculating space given by $\left\{\sum h_{\lambda \mu}^{a} e_{a}\right\}$.

Next if $u=(p, q)$ and $u^{\prime}=\left(p^{\prime}, q^{\prime}\right)$ then

$$
N\left(O_{\left(p, q^{\prime}\right)}^{1}(M)\right)=N\left(O_{(p, q)}^{1}(M)\right) \quad \text { and } \quad N\left(O_{\left(p^{\prime}, q^{\prime}\right)}^{1}(M)\right)=N\left(O_{\left(p, q^{\prime}\right)}^{1}(M)\right)
$$

which by the above means that every first osculating space to $\left.X\right|_{M}$ is perpendicular to every first osculating space to $\left.X\right|_{V}$. This in turn implies that the minimal enveloping subspaces $\mathscr{E}\left(M_{m}\right)$ and $\mathscr{E}\left(V_{n}\right)$ are perpendicular and this establishes the claim.

We apply this local criterion to give a proof of a theorem due to Moore [22].

THEOREM 9.3. Let $M_{m}$ be a connected Riemannian manifold with osculation degree $\rho_{1}\left(M_{m}\right) \equiv p_{1}$, and $V_{n}$ a connected Riemannian manifold with osculation 
degree $\rho_{1}\left(V_{n}\right) \equiv p_{2}$. Then any isometric immersion of the Riemannian product $X: M_{m} \times V_{n} \rightarrow R^{m+n+p_{1}+p_{2}}$ is substantial and reducible.

Proof. The hypothesis implies that we can choose product orthonormal frames in such a way that $\left(\Theta_{1}^{2}\right)^{p_{1}+1}=0,\left(\Theta_{1}^{2}\right)^{p_{1}} \neq 0$ and $\left(\Theta_{m+1}^{m+2}\right)^{p_{2}+1}=0$, $\left(\Theta_{m+1}^{m+2}\right)^{p_{2}} \neq 0$. Now by the Gauss equations and the fact that $\Theta_{\alpha}^{\lambda}=0$, we have

$$
\sum\left(\phi_{1}^{a}+\phi_{m+1}^{a}\right) \wedge\left(\phi_{2}^{a}+\phi_{m+2}^{a}\right)=\sum \alpha_{1}^{a} \wedge \phi_{2}^{a}+\sum \phi_{m+1}^{a} \wedge \phi_{m+2}^{a} .
$$

Since $\left(\Theta_{1}^{2}\right)^{p_{1}}$ and $\left(\Theta_{m+1}^{m+2}\right)^{p_{2}}$ are independently supported, the 2-form in (8.3) is of rank $p_{1}+p_{2}$ and $\left\{\phi_{1}^{a}+\phi_{m+1}^{a}, \phi_{2}^{a}+\phi_{m+2}^{a}\right\}$ are $2\left(p_{1}+p_{2}\right)$ linearly independent 1 -forms.

The curvature conditions $\Theta_{\beta}^{\lambda}=0$ and Cartan's lemma imply

$$
\phi_{1}^{b}, \phi_{m+1}^{b} \in\left\{\phi_{1}^{a}+\phi_{m+1}^{a}\right\}=V \text { and } \phi_{2}^{b}, \phi_{m+2}^{b} \in\left\{\phi_{2}^{a}+\phi_{m+2}^{a}\right\}=W,
$$

and hence equation (9.3) can be viewed in

$$
\Lambda^{2}(V \oplus W) \simeq \Lambda^{2} V \oplus V \otimes W \oplus \Lambda^{2} W
$$

where it becomes

$$
\sum\left(\phi_{1}^{a}+\phi_{m+1}^{a}\right) \otimes\left(\phi_{2}^{a}+\phi_{m+2}^{a}\right)=\Sigma \phi_{1}^{a} \otimes \phi_{2}^{a}+\sum \phi_{m+1}^{a} \otimes \phi_{m+2}^{a} .
$$

Now writing this equation in terms of the basis of $V \otimes W$ given by $\left(\phi_{1}^{a}+\phi_{m+1}^{a}\right) \otimes\left(\phi_{2}^{b}+\phi_{m+2}^{b}\right)$ we have matrices $A$ and $B$ defined by

$$
\sum \phi_{1}^{a} \otimes \phi_{2}^{a}=\sum A_{a b}\left(\phi_{1}^{a}+\phi_{m+1}^{a}\right) \otimes\left(\phi_{2}^{b}+\phi_{m+2}^{b}\right)
$$

and

$$
\sum \phi_{m+1}^{a} \otimes \phi_{m+2}^{a}=\sum B_{a b}\left(\phi_{1}^{a}+\phi_{m+1}^{a}\right) \otimes\left(\phi_{2}^{b}+\phi_{m+2}^{b}\right)
$$

which satisfy $\delta_{a b}=A_{a b}+B_{a b}$.

Now by Proposition 6.4 the minimal enveloping subspaces of the tensors

$$
-\Theta_{1}^{2}=\Sigma \phi_{1}^{a} \wedge \phi_{2}^{a} \text { and }-\Theta_{m+1}^{m+2}=\Sigma \phi_{m+1}^{a} \wedge \phi_{m+2}^{a}
$$

are orthogonal and hence $A B=B A=0$. Now if we choose a basis of the normal space adapted to the orthogonal decomposition $N=A N \oplus B N$, then $\phi_{1}^{a}, \phi_{2}^{a}$ are linearly independent and $\phi_{m+1}^{a}=\phi_{m+2}^{a}=0$ for the index range over $A N$, and $\phi_{1}^{a}=\phi_{2}^{a}=0$ and $\phi_{m+1}^{a}$ and $\phi_{m+2}^{a}$ are linearly independent for the index range over $B N$. As a result for $i=1,2$,

$$
0=-\Theta_{i}^{\lambda}=\Sigma \phi_{i}^{a} \wedge \phi_{\lambda}^{a},
$$

implies $\phi_{\lambda}^{a}=0$ for the index range over $A N$, and similarly $\phi_{\alpha}^{a}=0$ for the index range over $B N$. Together these last two vanishing statements imply $h_{\alpha \lambda}^{a}=0$ for the entire index range which establishes the local criterion of Proposition 9.1. 
An isometric immersion of a Riemannian product having an $m_{0}$-dimensional euclidean factor $X: M_{m} \times R^{m_{0}} \rightarrow R^{m+m_{0}+p}$ is called $m_{0}$-cylindrical (O'Neill [23]) if there exists an orthogonal decomposition $R^{m+m_{0}+p}=R^{m+p} \times R^{m_{0}}$ and an immersion $X_{M}: X_{M} \rightarrow R^{m+p}$ such that $X(u, v)=\left(X_{M}(u), v\right)$.

Our final application is to a theorem proved in codimension 2 by Alexander [3] and in arbitrary codimension by Moore [21].

TheOREM 9.4. Let $M_{i}, 1 \leqslant i \leqslant k$, be connected Riemannian manifolds with a nonzero sectional curvature at each point. Then an isometric immersion of the Riemannian product $X: M_{1} \times \cdots \times M_{k} \times R^{m_{0}} \rightarrow R^{N}$ where $N=\sum_{i=1}^{k} \operatorname{dim} M_{i}$ $+m_{0}+k$, is reducible and $m_{0}$-cylindrical.

Proof. The hypothesis on curvature implies that the curvature ranks $h_{i}$ of each $M_{i}$ are $\geqslant 2$. This implies the curvature deficiency $i$ of

$$
M_{1} \times \cdots \times M_{k} \times R^{m_{0}}
$$

is $\geqslant k$, but then the basic inequality of Theorem $6.7,0 \leqslant s-h \leqslant r-i$, implies $k \geqslant r \geqslant i \geqslant k$ and, hence, $r=i=k$ and $s=h$.

Now Proposition 7.1 implies $\beta$ is essential. Hence noting that

$$
\Phi^{0}\left(M_{1} \times \cdots \times M_{k} \times \mathbf{R}^{m_{0}}\right)=\Phi\left(R^{m_{0}}\right),
$$

we see that $\beta\left(\Phi, \Phi^{0}\right)=0$ now implies $\Phi\left(R^{m_{0}}\right)=\Phi^{0}=0$. Proposition 9.1 now implies that the euclidean factor is reducible, and Proposition 2.1 implies that the euclidean factor is a $m_{0}$-plane and hence the mapping is $m_{0}$-cylindrical. This reduces us to $M_{1} \times \cdots \times M_{k} \rightarrow R^{M}$ where $M=\sum_{i=1}^{k} \operatorname{dim} M_{i}+k$.

Since each $M_{i}$ has a nonzero sectional curvature, the curvature ranks $h_{i} \geqslant 2$, and this implies $\rho_{1}\left(M_{i}\right) \geqslant 1$. As such, Propositions 6.11 and 6.13 give

$$
k=r \geqslant \rho_{1}\left(M_{1} \times \cdots \times M_{k}\right)=\rho_{1}\left(M_{1}\right)+\cdots+\rho_{1}\left(M_{k}\right) \geqslant k .
$$

As a result $r=\rho_{1}\left(M_{1} \times \cdots \times M_{k}\right)$ and $\rho_{1}\left(M_{1}\right)=1$ and we may apply the last theorem inductively to split off the factors one at a time.

In [21] Moore gave a global version of this last reducibility theorem which drops the curvature requirements.

THEOREM. Let $M_{i}$ be compact connected Riemannian manifolds with

$$
\operatorname{dim} M_{i}=n_{i} \geqslant 2
$$

for $1 \leqslant i \leqslant p$, and let $M=M_{1} \times \cdots \times M_{p}$ be the Riemannian product. Then any isometric immersion $X: M \rightarrow R^{n_{1}+\cdots+n_{p}+p}$ is a product of hypersurface immersions.

This coupled with classical theorems on surfaces yields the following interesting example.

$$
X: \underbrace{S^{2} \times \cdots \times S^{2}}_{p} \rightarrow R^{3 p}
$$


is rigid. This is a genuine global theorem since local isometric deformations exist.

Additional global work on reducibility with other curvature assumptions and with compactness replaced by completeness may be found in [5], [16], [17], [19], [23], and [27].

\section{BIBLIOGRAPHY}

1. C. Allendoerfer, Global theorems in Riemannian geometry, Bull. Amer. Math. Soc. 54 (1948), 249-259. MR 10, 266.

2. - Rigidity for spaces of class greater than one, Amer. J. Math. 61 (1939), 633-644. MR 1, 28.

3. S. Alexander, Reducibility of Euclidean immersions of low codimension, J. Differential Geometry 3 (1969), 69-82. MR 40 \#3467.

4. _ Cylindricity of Euclidean immersions, Notices Amer. Math. Soc. 16 (1969), 682. Abstract \#69T-D16.

5. S. Alexander and R. Maltz, Isometric immersions of Riemannian products in Euclidean space, J. Differential Geometry (to appear).

6. W. Ambrose and I. Singer, A theorem on holonomy, Trans. Amer. Math. Soc. 75 (1953), 428-443. MR 16, 172.

7. G. M. Bergman, Ranks of tensors and change of base field, J. Algebra 11 (1969), 613-621. MR 39 \# 4177.

8. E. Cartan, Sur les variétés de courbure constante d'un espace euclidien ou non euclidien, Bull. Soc. Math. France 47 (1919), 125-160.

9. B. Chen, Geometry of submanifolds, Pure and Appl. Math., no. 22, Dekker, New York, 1973. MR 50 \# 5697.

10. S. S. Chern, La géométrie des sous-variétés d'un espace euclidien à plusieurs dimensions, Enseignement Math. 40 (1951-1954), 26-46. MR 16, 856.

11. - On curvature and characteristic classes of a Riemann manifold, Abh. Math. Sem. Univ. Hamburg 20 (1956), 117-126. MR 17, 783.

12. - On a theorem of algebra and its geometrical application, J. Indian Math. Soc. 8 (1944), 29-36. MR 6, 216.

13. S. S. Chern and N. H. Kuiper, Some theorems on the isometric imbedding of compact Riemann manifolds in Euclidean space, Ann. of Math. (2) 56 (1952), 422-430. MR 14, 408.

14. J. A. Erbacher, Reduction of the codimension of an isometric immersion, J. Differential Geometry 5 (1971), 333-340. MR 44 \#5897.

15. R. Gardner, Geometry of submanifolds of Euclidean spaces, Proc. Sympos. Pure Math., vol. 27, Amer. Math. Soc., Providence, R.I., 1975, pp. 125-134.

16. P. Hartman, On isometric immersions in Euclidean space of manifolds with non-negative sectional curvatures, Trans. Amer. Math. Soc. 115 (1965), 94-109. MR 34 \# 1968.

17. - On the isometric immersions in Euclidean space of manifolds with nonnegative sectional curvatures, II, Trans. Amer. Math. Soc. 147 (1970), 529-540. MR 41 \# 7586.

18. P. Hartman and $L$. Nirenberg, On spherical image maps whose Jacobians do not change sign, Amer. J. Math. 81 (1959), 901-920. MR 23 \# A4106.

19. N. H. Kuiper, Minimal total absolute curvature for immersions, Invent. Math. 10 (1970), 209-238. MR 42 \#2499.

20. R. Maltz, Cylindricity of isometric immersions into Euclidean space, Proc. Amer. Math. Soc. 53 (1975), 428-432.

21. J. D. Moore, Isometric immersions of Riemannian products, J. Differential Geometry 5 (1971), 159-168. MR 46 \#6249.

22. - Reducibility of isometric immersions, Proc. Amer. Math. Soc. 34 (1972), 229-232. MR 45 \#2623.

23. B. O'Neill, Isometric immersion of flat manifolds in euclidean space, Michigan Math. J. 9 (1962), 199-205. MR 27 \#2941.

24. F. A. E. Pirani, Invariant formulation of gravitational radiation theory, Phys. Rev. (2) 105 (1957), 1089-1099. MR 20 \#3020. 
25. M. Spivak, A comprehensive introduction to differential geometry. Vols. I-V, Publish or Perish, Boston, Mass., 1970-75. MR 42 \#2369; \#6726.

26. S. Sternberg, Lectures on differential geometry, Prentice-Hall, Englewood Cliffs, N.J., 1964. MR 33 \# 1797.

27. Edsel Stiel, Isometric immersions of manifolds of nonnegative constant sectional curvature, Pacific J. Math. 15 (1965), 1415-1419. MR 32 \#6375.

28. C. Tompkins, Isometric imbedding of flat manifolds in Euclidean space, Duke Math. J. 5 (1939), 58-61.

29. H. Weyl, On the volume of tubes, Amer. J. Math. 61 (1939), 461-472.

30. K. Yano and S. Bochner, Curvature and Betti numbers, Ann. of Math. Studies, no. 32 , Princeton Univ. Press, Princeton, N.J., 1953. MR 15, 989.

Department of Mathematics, University of North Carolina, Chapel Hill, North CAROLINA 27514 